\title{
A power flow mode theory based on a system's damping distribution and power flow design approaches
}

\author{
By Ye-Ping Xiong ${ }^{1,2}$, J. T. Xing ${ }^{1}$ and W. G. Price ${ }^{1}$ \\ ${ }^{1}$ School of Engineering Sciences, Ship Science, University of Southampton, \\ Southampton SO17 1BJ, UK \\ (j.t.xing@ship.soton.ac.uk) \\ ${ }^{2}$ Institute of Engineering Mechanics, Shandong University, Jinan 250061, \\ People's Republic of China
}

A power flow mode theory is developed to describe the natural power flow behaviour of a dynamic system based on its inherent damping distribution. The system's characteristicdamping matrix is constructed and it is shown that the eigenvalues and eigenvectors of this matrix identify natural power flow characteristics. These eigenvectors, or power flow mode vectors, are chosen as a set of base-vectors spanning the power flow space and completely describe the power flow in the system. The generalized coordinate of the velocity vector decomposed in this space defines the power flow response vector. A timeaveraged power flow expression and theorems relating to its estimation are presented.

Based on this theory, power flow design approaches are proposed to identify energy flow patterns satisfying vibration control requirements. The mode control factor defines the measure of the correlation between a power flow mode and a natural vibration mode of the system. Power flow design theorems are presented providing guidelines to construct damping distributions maximizing power dissipation or to suppress/retain a particular vibration mode and/or a motion.

The developed damping-based power flow mode theory is compared with a mobilitybased power flow model. It is shown that the proposed power flow model provides insight into the power flow dissipation mechanisms in dynamic systems.

Examples are presented to demonstrate the applicability of the power flow mode theory and the power flow design approach. These examples demonstrate the generality of the theory, including non-symmetric damping matrices, and illustrate power flow design applications through modifications of the system's damping distribution using passive and/or active control components.

Keywords: power flow mode theory; power flow space; power flow mode vector; characteristic-damping factor; natural power flow characteristics; power flow design

\section{Introduction}

A power flow analysis provides a technique to describe the dynamic behaviour of complex structures and coupled systems at medium to high values of frequency. The approach focuses on the flow of vibrational energy rather than the detailed 
spatial pattern of the structural response. The fundamental concept of power flow is discussed by Goyder \& White (1980) and Pinnington \& White (1981). In recent years, this approach has been applied to model complex structures (Cuschieri 1990; Langley 1990; Xing \& Price 1999; Xiong et al. 2001, 2003a), structural-acoustic problems (Petersson \& Plunt 1982; Su et al. 1995) and to assess passive, active or hybrid vibration control systems (Miller et al. 1990; Pan et al. 1992; Xiong 1996; Xiong et al. 2000). To predict input power and power transmission in a system requires knowledge of the excitations (forces or motions) and dynamic responses (motions or forces) derived, for example, by analytical, numerical or experimental mobility or impedance approaches, travelling wave methods, etc. (see, for example, Price \& Keane 1994; Fahy \& Price 1999).

Based on the conventional modal decomposition technique described by Timoshenko et al. (1974), Pinnington (1986) concluded that the total input power equals the sum of the input powers to each vibration mode. Later, $\mathrm{Su}$ et al. (1995) developed a power flow expression using the eigenvalues and the corresponding eigenvectors of the real part of the mobility matrix. The concept and formulation of the upper and lower bounds of power flows were presented by $\mathrm{Su}$ et al. (1995), which was also investigated by Moorhouse (2002). These contributions laid the foundation for the mobility-based power flow mode approach proposed by Ji et al. (2003). This method uses the eigenproperties of the real part of the mobility matrix and power mode force vector to describe the time-averaged power input of the excitation forces applied to a system. However, to predict input power and power transmission by this method, full knowledge of the system's mobility is required to determine its eigenproperties. This mobility matrix depends on all the system's physical properties including inertia, elastic and damping parameters as well as the frequency of the external forces.

A similar dependence was found relating to sound power radiation from structures. For example, in references cited by Fuller et al. (1997), the application of mode superposition method and wavenumber Fourier transformation provided the sound power output from a plate $\Pi=\dot{\boldsymbol{w}}^{\mathrm{H}} \boldsymbol{M} \dot{\boldsymbol{w}}$, where $\dot{\boldsymbol{w}}$ is the generalized velocity vector corresponding to the $N$ chosen natural mode functions of the plate and $M$ is a matrix which has its eigenvalues $\Omega_{n}$ and eigenvictors $P_{n}(n=1,2,3, \ldots, N)$. Based on analysis, this was shown to be expressible as $\Pi=\sum_{n=0}^{N} \Omega_{n}\left|b_{n}\right|^{2}$, where $b_{n}=\boldsymbol{P}_{n} \dot{\boldsymbol{w}}$. Here, again, the matrix $\boldsymbol{M}$ is dependent of the frequency of excitation, wavenumbers and natural mode functions of the plate, and therefore involves external excitations, stiffness and mass parameters of the system.

As is well known, for harmonic motions or stationary random processes, the kinetic and potential energies remain unchanged after every cycle (Price \& Bishop 1974; Newland 1975), from which it is concluded that the time-averaged power of a system equals the energy dissipated by its damping (Xing \& Price 1999). Therefore, in theory, the natural characteristics of power flow should be defined by the damping property of the system. This paper addresses this problem through the development of a power flow mathematical model based on the inherent damping characteristics of the system.

From this idea, a damping-based power flow mode theory is developed to describe the essential power flow behaviour of a dynamic system. A characteristic-damping matrix is introduced from physical damping matrix components. The eigenvalues and eigenvectors of this characteristic-damping matrix are defined as the characteristic-damping factor and the power flow mode vectors of the system, 
respectively, and these parameters are independent of external excitations. Power flow mode vectors are chosen as a set of base-vectors spanning the power flow space to describe completely the system's power flow behaviour. The generalized coordinate of the velocity vector decomposed in the power flow space is defined as the power flow response vector, which depends on external vibration sources. The time-averaged power flow is determined by the characteristic-damping factor and the power flow response vector. For a unit power flow response vector, the time-averaged power flow value depends only on the system's characteristic-damping factor.

Based on this theory and the power flow design concept discussed by Xing et al. (2002), power flow design theorems are proposed on which design approaches are developed and demonstrated. Examples illustrating the generality of the proposed mathematical model, theory, design procedure, etc. are presented as well as applications for vibration analysis and control.

\section{Generalized formulation of a dynamic system}

For generality, consider the dynamics of a generalized linear system with $N$ degrees-of-freedom described by the matrix equation

$$
\begin{gathered}
M \ddot{\tilde{\boldsymbol{X}}}+\boldsymbol{C} \dot{\tilde{\boldsymbol{X}}}+\boldsymbol{K} \tilde{\boldsymbol{X}}=\tilde{\boldsymbol{f}} \mathrm{e}^{\mathrm{i} \omega t}, \\
C=C_{\mathrm{v}}+C_{\mathrm{h}},
\end{gathered}
$$

where $\tilde{\boldsymbol{f}}$ denotes the complex amplitude of the excitation force vector, $\boldsymbol{M}$ is a real, symmetric and semi-positive definite mass matrix, $\boldsymbol{K}$ represents a symmetric and semi-positive definite stiffness matrix and $\boldsymbol{C}$ is a damping matrix consisting of a viscous part $\boldsymbol{C}_{\mathrm{v}}$ and a hysteresis component $\boldsymbol{C}_{\mathrm{h}}=(\eta / \omega) \overrightarrow{\boldsymbol{C}}_{\mathrm{h}}$. Here, $\eta$ represents a loss factor and $\overline{\boldsymbol{C}}_{\mathrm{h}}$ is a real symmetric matrix relating to the subsystems in which hysteresis damping exists. Generally, $\overline{\boldsymbol{C}}_{\mathrm{h}}$ is not the stiffness matrix $\boldsymbol{K}$ of the system and in general $\boldsymbol{C}$ may be non-symmetric because of active damping contributions arising from different sources of velocity feedback gains (Xiong et al. 2003b; Xing et al. 2005). The matrices $\tilde{\boldsymbol{X}}, \dot{\tilde{\boldsymbol{X}}}$ and $\tilde{\tilde{\boldsymbol{X}}}$ represent the displacement, velocity and acceleration vectors of the system, respectively. For cases of motion excitation, the external force may vanish, i.e. $\tilde{f}=0$, but the motions of some degrees of freedom of the system are prescribed.

The solution of equation $(2.1 a)$ and $(2.1 b)$ takes the form $\dot{\tilde{X}}=\tilde{\boldsymbol{V}} \mathrm{e}^{\mathrm{i} \omega t}$, which allows equation $(2.1 a)$ and $(2.1 b)$ to be expressed as

$$
\begin{gathered}
\tilde{f}=\tilde{Z} \tilde{V}, \\
\tilde{V}=\tilde{Y} \tilde{f},
\end{gathered}
$$

provided the inverse matrix $\tilde{\boldsymbol{Z}}^{-1}$ exists. Here $\tilde{\boldsymbol{V}}$ is a velocity amplitude vector of the system, $\tilde{\boldsymbol{Z}}$ denotes the impedance matrix

$$
\tilde{Z}=\mathrm{i} \omega \boldsymbol{M}+\boldsymbol{C}+\frac{\boldsymbol{K}}{\mathrm{i} \omega},
$$

and $\tilde{\boldsymbol{Y}}$, the system's mobility matrix, is defined by

$$
\tilde{\boldsymbol{Y}}=\tilde{Z}^{-1}
$$


such that $\tilde{\boldsymbol{Y}} \tilde{\boldsymbol{Z}}=\boldsymbol{I}=\tilde{\boldsymbol{Z}} \tilde{\boldsymbol{Y}}$, where $\boldsymbol{I}$ denotes a unit matrix. In general, the complex matrices $\tilde{\boldsymbol{Y}}$ and $\tilde{\boldsymbol{Z}}$ are non-symmetric because of the inherent nature of the system.

To aid discussion, it is assumed that $\boldsymbol{X}=\left[\boldsymbol{\chi}_{1}, \boldsymbol{\chi}_{2}, \ldots, \boldsymbol{\chi}_{N}\right]$ represents the matrix of normalized natural vibration modes satisfying the following orthogonal relations (Bishop \& Price 1979)

$$
\begin{gathered}
\boldsymbol{X}^{\mathrm{T}} \boldsymbol{X}=\boldsymbol{I}, \\
\boldsymbol{X}^{\mathrm{T}} \boldsymbol{M} \boldsymbol{X}=\operatorname{diag}\left(M_{j}\right), \\
\boldsymbol{X}^{\mathrm{T}} \boldsymbol{K} \boldsymbol{X}=\operatorname{diag}\left(K_{j}\right), \\
\omega_{j}^{2}=K_{j} / M_{j},
\end{gathered}
$$

where $\omega_{j}^{2}$ denotes the $j$ th natural frequency of the system.

\section{Power flow mode theory}

\section{(a) Characteristic-damping matrix}

The total time-averaged power input by all excitation forces applied to a system (Skudrzyk 1968) is given by the expression

$$
P=\frac{1}{2} \operatorname{Re}\left(\tilde{\boldsymbol{f}}^{\mathrm{H}} \tilde{\boldsymbol{V}}\right)=\frac{1}{2} \operatorname{Re}\left(\tilde{\boldsymbol{V}}^{\mathrm{H}} \tilde{\boldsymbol{f}}\right)=\frac{1}{4} \operatorname{Re}\left(\tilde{\boldsymbol{V}}^{\mathrm{H}} \tilde{\boldsymbol{f}}+\tilde{\boldsymbol{f}}^{\mathrm{H}} \tilde{\boldsymbol{V}}\right),
$$

where the superscript ' $\mathrm{H}$ ' denotes a conjugate, transpose matrix.

Taking the conjugate transpose of equation (2.2), we find that

$$
\tilde{\boldsymbol{V}}^{\mathrm{H}}\left(-\mathrm{i} \omega \boldsymbol{M}^{\mathrm{T}}+\boldsymbol{C}^{\mathrm{T}}-\frac{\boldsymbol{K}^{\mathrm{T}}}{\mathrm{i} \omega}\right)=\tilde{\boldsymbol{f}}^{\mathrm{H}},
$$

where superscript ' $\mathrm{T}$ ' denotes a transpose matrix. By continuing this matrix manipulation by pre-multiplying equation $(2.2)$ by $\tilde{\boldsymbol{V}}^{\mathrm{H}}$ and post-multiplying equation (3.2) by $\tilde{\boldsymbol{V}}$, we derive the results

$$
\begin{gathered}
\tilde{\boldsymbol{V}}^{\mathrm{H}}\left(\mathrm{i} \omega \boldsymbol{M}+\boldsymbol{C}+\frac{\boldsymbol{K}}{\mathrm{i} \omega}\right) \tilde{\boldsymbol{V}}=\tilde{\boldsymbol{V}}^{\mathrm{H}} \tilde{\boldsymbol{f}}, \\
\tilde{\boldsymbol{V}}^{\mathrm{H}}\left(-\mathrm{i} \omega \boldsymbol{M}^{\mathrm{T}}+\boldsymbol{C}^{\mathrm{T}}-\frac{\boldsymbol{K}^{\mathrm{T}}}{\mathrm{i} \omega}\right) \tilde{\boldsymbol{V}}=\tilde{\boldsymbol{f}}^{\mathrm{H}} \tilde{\boldsymbol{V}} .
\end{gathered}
$$

Since $\boldsymbol{M}^{\mathrm{T}}=\boldsymbol{M}$ and $\boldsymbol{K}^{\mathrm{T}}=\boldsymbol{K}$, a summation of equations (3.3) and (3.4) gives

$$
\tilde{\boldsymbol{V}}^{\mathrm{H}}\left(\boldsymbol{C}+\boldsymbol{C}^{\mathrm{T}}\right) \tilde{\boldsymbol{V}}=\tilde{\boldsymbol{V}}^{\mathrm{H}} \tilde{\boldsymbol{f}}+\tilde{\boldsymbol{f}}^{\mathrm{H}} \tilde{\boldsymbol{V}} .
$$

From equations (3.1) and (3.5), Xiong et al. (2004) showed that after matrix manipulation the time-averaged input power takes the form

where

$$
P=\frac{1}{2} \operatorname{Re}\left\{\tilde{\boldsymbol{V}}^{\mathrm{H}} \overline{\boldsymbol{C}} \tilde{\boldsymbol{V}}\right\},
$$

$$
\overline{\boldsymbol{C}}=\frac{1}{2}\left(\boldsymbol{C}+\boldsymbol{C}^{\mathrm{T}}\right) .
$$

This result demonstrates that the time-averaged power of a system equals the power dissipated by damping, which is consistent with the result derived from 
the general theory of continuum mechanics developed by Xing \& Price (1999). For a simple system with one-degree-of-freedom of damping coefficient $c$ and velocity $v$, this reduces to $P=(1 / 2) c|v|^{2}$.

Because $\boldsymbol{C}+\boldsymbol{C}^{\mathrm{T}}$ is a real symmetric matrix, it follows that $\overline{\boldsymbol{C}}$ is a real symmetric matrix allowing exploration of the properties of real symmetric matrices. In general, $\bar{C}$ is a semi-positive definite matrix if there exists no negative active damping in the system. As is subsequently shown, this characteristic-damping matrix $\bar{C}$ plays a significant role in determining the system's power flow.

\section{(b) Power flow space}

Based on matrix theory (Nering 1963), the characteristic-damping matrix $\overline{\boldsymbol{C}}$ decomposes into

$$
\bar{C}=\Phi \Lambda \Phi^{\mathrm{T}}
$$

where

$$
\begin{gathered}
\boldsymbol{\Lambda}=\operatorname{diag}\left(\lambda_{j}\right), \\
\boldsymbol{\Phi}=\left[\boldsymbol{\varphi}_{1}, \boldsymbol{\varphi}_{2}, \ldots, \boldsymbol{\varphi}_{N}\right], \quad \boldsymbol{\Phi}^{\mathrm{T}} \boldsymbol{\Phi}=\boldsymbol{\Phi} \boldsymbol{\Phi}^{\mathrm{T}}=\boldsymbol{I} .
\end{gathered}
$$

Here $\lambda_{j}$, of dimension $\mathrm{Ns} \mathrm{m}^{-1}$, and non-dimensional real vectors $\boldsymbol{\varphi}_{j}(j=1$, $2, \ldots, N)$, denote the eigenvalues and the corresponding eigenvectors of the characteristic-damping matrix $\overline{\boldsymbol{C}}$, respectively. These power flow mode vectors are linearly independent of each other and they are chosen as a set of base vectors spanning the power flow space of the system.

\section{(c) Natural power flow characteristics}

The $j$ th characteristic-damping factor $\lambda_{j}$ and $j$ th power flow mode vector $\boldsymbol{\varphi}_{j}$ of the characteristic-damping matrix $\overline{\boldsymbol{C}}$ are independent of external excitations. For a dynamic system, the characteristic-damping matrix $\bar{C}$ is prescribed and accordingly its natural power flow characteristics, i.e. $\lambda_{j}$ and $\boldsymbol{\varphi}_{j}$, are determined.

\section{(d) Power flow response vector}

In the defined power flow space, the physical velocity vector $\tilde{\boldsymbol{V}}$ of the system decomposes into the form

$$
\tilde{\boldsymbol{V}}=\boldsymbol{\Phi} \tilde{\boldsymbol{q}}
$$

Since $\boldsymbol{\Phi}$ is the orthogonal matrix composed of the corresponding eigenvectors $\boldsymbol{\varphi}_{j}$ $(j=1,2, \ldots, N)$, we obtain

$$
\begin{gathered}
\tilde{\boldsymbol{q}}=\boldsymbol{\Phi}^{\mathrm{T}} \tilde{\boldsymbol{V}}, \\
\tilde{q}_{j}=\boldsymbol{\varphi}_{j}^{\mathrm{T}} \tilde{\boldsymbol{V}},
\end{gathered}
$$

which defines a complex valued velocity vector of dimension $\mathrm{ms}^{-1}$. For a prescribed system, this power flow response vector $\tilde{\boldsymbol{q}}$ defined in the power flow space depends on the external excitations and its $j$ th element $\tilde{q}_{j}$ denotes the $j$ th power flow response corresponding to the $j$ th power flow mode $\boldsymbol{\varphi}_{j}$. 


\section{(e) Power flow expression}

A substitution of equations $(3.8 a-c)$ and (3.9) into equation (3.6) produces an expression for the time-averaged power flow in the form

where

$$
P=\frac{1}{2} \operatorname{Re}\left\{\tilde{\boldsymbol{q}}^{\mathrm{H}} \boldsymbol{\Lambda} \tilde{\boldsymbol{q}}\right\}=\frac{1}{2} \sum_{j=1}^{N} \lambda_{j}\left|\tilde{q}_{j}\right|^{2}=\sum_{j=1}^{N} P_{j},
$$

$$
\begin{gathered}
P_{j}=\lambda_{j}\left|\tilde{q}_{j}\right|^{2} / 2, \\
P_{j} /\left|\tilde{q}_{j}\right|^{2}=\lambda_{j} / 2 \quad(j=1,2, \ldots, N),
\end{gathered}
$$

defines the $j$ th mode dissipation power that represents the $j$ th time-averaged power flow component of the system. These expressions are summarized in the following theorem.

Power flow expression theorem 3.1. For a dynamic system described by equation (2.1a,b), its time averaged power flow equals the sum of all mode dissipation power components $P_{j}=\lambda_{j}\left|\tilde{q}_{j}\right|^{2} / 2$ determined by the characteristicdamping factor $\lambda_{j}$ and characteristic velocity $\tilde{q}_{j}$.

The system's total time-averaged power flow comprises of the power dissipated by all $N$ independent power flow modes, each relating only to its characteristic-damping factor and power flow response vector. It also provides assessment of the role of damping in power dissipation. For example, for unit characteristic velocities $\tilde{q}_{j}$, the time-averaged power flow component $P_{j}$ depends only on the system's natural characteristic-damping factor $\lambda_{j}$, which is independent of all external forces. A large value of $\lambda_{j}$ implies significant energy dissipation in the $j$ th power flow mode $\boldsymbol{\varphi}_{j}$.

\section{(f) Power flow bounds}

Let us assume that $\lambda_{1}$ and $\lambda_{N}$ denote the smallest and largest eigenvalues of the characteristic-damping matrix $\overline{\boldsymbol{C}}$. The time-averaged power $P$ can be estimated to lie in the range defined by the lower bound $P_{\text {low }}$ and the upper bound $P_{\mathrm{up}}$ as given by

$$
P_{\text {low }}=\frac{1}{2}\left(\sum_{j=1}^{N}\left|\tilde{q}_{j}\right|^{2}\right) \lambda_{1}<P<\frac{1}{2}\left(\sum_{j=1}^{N}\left|\tilde{q}_{j}\right|^{2}\right) \lambda_{N}=P_{\text {up }} .
$$

Noting that $\tilde{\boldsymbol{q}}^{\mathrm{H}} \tilde{\boldsymbol{q}}=\tilde{\boldsymbol{V}}^{\mathrm{H}} \boldsymbol{\Phi} \boldsymbol{\Phi}^{\mathrm{T}} \tilde{\boldsymbol{V}}=\tilde{\boldsymbol{V}}^{\mathrm{H}} \tilde{\boldsymbol{V}}$, it follows that

$$
\sum_{j=1}^{N}\left|\tilde{q}_{j}\right|^{2}=\sum_{j=1}^{N}\left|\tilde{v}_{j}\right|^{2}
$$

and we can rewrite equation (3.13) in the form

$$
P_{\text {low }}=\frac{1}{2}\left(\sum_{j=1}^{N}\left|\tilde{v}_{j}\right|^{2}\right) \lambda_{1}<P<\frac{1}{2}\left(\sum_{j=1}^{N}\left|\tilde{v}_{j}\right|^{2}\right) \lambda_{N}=P_{\text {up }} .
$$

Dividing this equation by the square of the velocity vector modulus, i.e. $|\tilde{\boldsymbol{V}}|^{2}=\sum_{j=1}^{N}\left|\tilde{v}_{j}\right|^{2}$, we find that

$$
\frac{\lambda_{1}}{2}<\frac{P}{|\tilde{\boldsymbol{V}}|^{2}}<\frac{\lambda_{N}}{2} .
$$


Physically, $P /|\tilde{\boldsymbol{V}}|^{2}$ represents the power dissipated per unit square of velocity vector modulus. This result is summarized as follows.

Power flow bounds theorem 3.2. For a dynamic system defined by equation (2.1a,b), its time averaged power dissipation $P /|\tilde{\boldsymbol{V}}|^{2}$ per unit square of velocity vector modulus is bounded by one half of the lowest and highest characteristicdamping factor values of the system.

Equation (3.16) provides an estimation of the time-averaged power flow in terms of the maximum and minimum characteristic-damping factors and these relate to the damping of the system only and do not require any information of vibration sources and responses of the system. It provides a measure to assess and compare the power dissipation capabilities of dynamic systems. Based on this theorem, the following corollaries are derived.

(i) Regardless of the external excitations and mass/stiffness distributions, the power dissipated per unit square norm of velocity vector $P /|\tilde{\boldsymbol{V}}|^{2}$ is always bounded by one half of the maximum and minimum characteristic-damping factor values.

(ii) For any system modelled by equation $(2.1 a, b)$ and having the same damping matrix $\boldsymbol{C}$ but different stiffness and mass distributions as well as different external forces, the power dissipated per unit square of velocity vector modulus $P /|\tilde{\boldsymbol{V}}|^{2}$ of all such systems are bounded by the same upper and lower bound values given in equation (3.16).

(iii) Any modification of the stiffness and mass matrices, natural frequencies and external excitations in equation $(2.1 a, b)$ do not change the bounds of $P /|\tilde{\boldsymbol{V}}|^{2}$ given in equation (3.16) provided the damping matrix of the system $\boldsymbol{C}$ remains unaffected.

\section{Power flow design}

The power flow mode theory developed in $\$ 3$ establishes a theoretical basis for a power flow design approach which aims to identify various appropriate power flow patterns through designed damping distributions. Based on the presented theory, three power flow design theorems are expressed as follows.

\section{(a) Maximum power dissipation}

Theorem 4.1. For a dynamic system defined by equation (2.1a,b), the time averaged power dissipated per unit characteristic velocity $\tilde{q}_{j}(j=1,2,3, \ldots, N)$ is maximum if and only if the trace of its characteristic-damping matrix is a maximum value.

Proof. On using equation (3.11), we find that the time-averaged power dissipated per unit characteristic velocity of the system is given by

$$
P_{\mathrm{u}}=\left(\lambda_{1}+\lambda_{2}+\cdots+\lambda_{N}\right) / 2 \text {. }
$$

Based on matrix theory (Nering 1963), the orthogonal transformation represented by equation (3.8a) does not change the trace of the real symmetric matrix $\overline{\boldsymbol{C}}$ and therefore

$$
P_{\mathrm{u}}=\left(\lambda_{1}+\lambda_{2}+\cdots+\lambda_{N}\right) / 2=\operatorname{tr} \bar{C} / 2 .
$$


This equation demonstrates that the maximum $P_{\mathrm{u}}$ and $\operatorname{tr} \overline{\boldsymbol{C}}$ of the system are equivalent quantities and therefore the theorem is valid.

Theorem 4.2. If a dynamic system defined by equation (2.1) dissipates the maximum time averaged power for any external force vector $\tilde{\boldsymbol{f}} \mathrm{e}^{\mathrm{i} \omega t}$, the trace of its characteristic-damping matrix is maximum.

Proof. If the condition expressed in theorem 4.2 is valid, for any external force vector $\tilde{\boldsymbol{f}} \mathrm{e}^{\mathrm{i} \omega t}$ the time averaged power dissipation represented by equation (3.11) should be maximum. Now, we can find an external force vector by letting $\left|\tilde{q}_{j}\right|=1(j=1,2,3, \ldots, N)$ and using equations $(2.1 a, b)$ and $(3.9)$ it follows that

$$
\tilde{\boldsymbol{f}} \mathrm{e}^{\mathrm{i} \omega t}=j \omega \boldsymbol{M} \tilde{\boldsymbol{V}}+\boldsymbol{C} \tilde{\boldsymbol{V}}+\frac{1}{j \omega} \boldsymbol{K} \tilde{\boldsymbol{V}}=\left(j \omega \boldsymbol{M}+\boldsymbol{C}+\frac{1}{j \omega} \boldsymbol{K}\right) \boldsymbol{\Phi} \tilde{\boldsymbol{q}}^{*},
$$

where $\tilde{\boldsymbol{q}}^{*}=[1,1,1, \ldots, 1]^{\mathrm{T}}$. The time averaged power dissipation corresponding to the force vector given by equation (4.3) satisfies equation (4.2) which is also maximum according to the condition of theorem 4.1 and therefore theorem 4.2 is valid. The latter also provides the necessary condition on which to design a damping distribution to obtain the maximum average power dissipated in the system.

\section{(b) Power flow mode design for a natural vibration mode}

The velocity vector $\tilde{\boldsymbol{V}}$ can be decomposed in the vibration mode space, defined by the natural mode vectors given in equation $(2.5 a-d)$, into the form

$$
\tilde{\boldsymbol{V}}=\boldsymbol{X} \tilde{\boldsymbol{R}}
$$

where $\boldsymbol{X}=\left[\boldsymbol{\chi}_{1}, \boldsymbol{\chi}_{2}, \ldots, \boldsymbol{\chi}_{N}\right]$ is the normalized natural vibration modes of a system, and $\tilde{\boldsymbol{R}}$ is a general velocity vector. Substituting equation (4.4) into equation $(3.10 a, b)$ we find that

and

$$
\tilde{\boldsymbol{q}}=\boldsymbol{\Phi}^{\mathrm{T}} \boldsymbol{X} \tilde{\boldsymbol{R}}
$$

$$
\tilde{q}_{j}=\sum_{i=1}^{N} \boldsymbol{\varphi}_{j}^{\mathrm{T}} \boldsymbol{\chi}_{i} \tilde{R}_{i}=\sum_{i=1}^{N} \boldsymbol{\gamma}_{j i} \tilde{R}_{i}
$$

where the mode control factor is defined as

$$
\gamma_{j i}=\boldsymbol{\varphi}_{j}^{\mathrm{T}} \boldsymbol{\chi}_{i} \quad(i, j=1,2,3, \ldots, N) .
$$

This is a dot product of the $i$ th natural vibration mode and $j$ th power flow mode. Physically, the mode control factor $\gamma_{j i}$ represents the $i$ th natural mode component of the characteristic velocity $\tilde{q}_{j}$.

On using equations (3.11) and (4.6), the energy dissipated in the pure (i.e. only $\left.\tilde{R}_{i} \neq 0\right) i$ th vibration mode $\boldsymbol{\chi}_{i}$ is expressed as

$$
P_{i}=\frac{1}{2} \sum_{j=1}^{N} \lambda_{j}\left|\gamma_{j i}\right|^{2}\left|\tilde{R}_{i}\right|^{2} .
$$

The energy dissipated per unit square of the $i$ th generalized velocity of the $i$ th natural mode is given by

$$
P_{i} /\left|\tilde{R}_{i}\right|^{2}=\frac{1}{2} \sum_{j=1}^{N} \lambda_{j}\left|\gamma_{j i}\right|^{2} .
$$


These two expressions relate the energy dissipation of the $i$ th pure vibration mode to the power flow modes in the power flow mode space. A large value of $\left|\gamma_{j i}\right|$ represents a large component of $\tilde{q}_{j}$ produced by the $i$ th natural vibration mode and therefore a large energy dissipation $(1 / 2) \lambda_{j}\left|\gamma_{j i}\right|^{2}$ in the $j$ th power flow mode. The suppression of the $i$ th vibration mode can be realized by designing a power flow mode $\boldsymbol{\varphi}_{j}$ that provides a large absolute value of mode control factor $\gamma_{j i}$. In the reverse case, to retain the $i$ th vibration mode, we need to design power flow mode $\boldsymbol{\varphi}_{j}$ that provides a zero mode control factor $\gamma_{j i}$. These findings can be summarized in the following power flow mode design theorems.

Theorem 4.3. If a power flow mode $j$ is designed orthogonal to a natural vibration mode $i$, i.e. $\gamma_{j i}=0$, there is no power dissipated by the ith natural vibration mode in the power flow mode $j$ and therefore the ith natural vibration mode is not damped by the jth power flow mode.

Theorem 4.4. If a power flow mode $j$ with a positive characteristic factor $\lambda_{j}$ is designed parallel to a natural vibration mode $i$, that is, $\gamma_{j i}$ has a maximum absolute value, there is a maximum of energy dissipated by the ith natural vibration mode in the power flow mode $j$ and therefore the ith natural vibration mode is effectively damped by the jth power flow mode.

\section{(c) Power flow mode design for a prescribed motion}

From equations $(3.10 a, b)$ and $(3.12 a, b)$, we conclude the following power flow mode design theorems for a prescribed motion.

Theorem 4.5. If a power flow mode $j$ is designed orthogonal to a prescribed motion $\tilde{\boldsymbol{V}}$, i.e. $\tilde{q}_{j}=0$, there is no power dissipated by this motion in the power flow mode $j$ and therefore this prescribed motion is not damped by the jth power flow mode.

Theorem 4.6. If a power flow mode $j$ with a positive characteristic factor $\lambda_{j}$ is designed parallel to a prescribed motion $\tilde{\boldsymbol{V}}$, i.e. $\tilde{q}_{j}$ has a maximum absolute value, there is a maximum of energy dissipated by this motion in the power flow mode $j$ and therefore this prescribed motion is effectively damped by the jth power flow mode.

\section{Comparison with mobility-based power flow model}

\section{(a) Generalization of the mobility-based power flow mode approach}

A mobility-based power flow mode approach was proposed by Su et al. (1995) and extended by Ji et al. (2003) assuming a symmetric mobility matrix of the system. This assumption is valid for many cases. However, the real part of the mobility matrix of an active velocity feedback control system may become non-symmetric as demonstrated by Xiong et al. (2003b). Therefore, in this paper the requirement of the symmetric real part of a mobility matrix is released. In general, the symmetry of the imaginary part of the mobility matrix involving mass and stiffness matrices is valid. Based on the above description, the generalization of the mobility-based power flow mode approach is presented as follows.

The time-averaged power flow in a mobility formulation is given by

$$
P=\frac{1}{4} \operatorname{Re}\left\{\tilde{\boldsymbol{f}}^{\mathrm{H}}\left(\tilde{\boldsymbol{Y}}+\tilde{\boldsymbol{Y}}^{\mathrm{H}}\right) \tilde{\boldsymbol{f}}\right\}=\frac{1}{2} \operatorname{Re}\left\{\tilde{\boldsymbol{f}}^{\mathrm{H}} \overline{\boldsymbol{Y}} \tilde{\boldsymbol{f}}\right\},
$$


where

$$
\overline{\boldsymbol{Y}}=\frac{1}{2}\left(\tilde{\boldsymbol{Y}}+\tilde{\boldsymbol{Y}}^{\mathrm{H}}\right)=\frac{1}{2}\left\{\operatorname{Re}(\tilde{\boldsymbol{Y}})+\operatorname{Re}\left(\tilde{\boldsymbol{Y}}^{\mathrm{H}}\right)\right\}
$$

is the real, symmetric characteristic mobility matrix of the system. If the symmetry of the real part of the mobility matrix is assumed, it is obtained that $\left(\tilde{\boldsymbol{Y}}+\tilde{\boldsymbol{Y}}^{\mathrm{H}}\right)=2 \operatorname{Re}(\tilde{\boldsymbol{Y}})$ as presented by Ji et al. (2003). This matrix $\overline{\boldsymbol{Y}}$ can be decomposed into the form

$$
\overline{\boldsymbol{Y}}=\boldsymbol{\Psi} \boldsymbol{\Xi} \boldsymbol{\Psi}^{\mathrm{T}},
$$

where $\boldsymbol{\Xi}$ is a real diagonal matrix of the eigenvalues $\mu_{j}$ of the characteristic mobility matrix $\overline{\boldsymbol{Y}}$ and $\boldsymbol{\Psi}=\left[\psi_{1}, \psi_{2}, \ldots, \psi_{N}\right]$ is a corresponding orthogonal matrix of eigenvectors satisfying

$$
\boldsymbol{\Psi} \boldsymbol{\Psi}^{\mathrm{T}}=\boldsymbol{\Psi}^{\mathrm{T}} \boldsymbol{\Psi}=\boldsymbol{I} .
$$

Here the $j$ th characteristic mobility factor $\mu_{j}$ has dimension $\mathrm{m}(\mathrm{Ns})^{-1}$ of mobility. Decomposing the force vector $\tilde{\boldsymbol{f}}$ in the power flow space defined by $\boldsymbol{\Psi}$, we obtain

and

$$
\tilde{f}=\boldsymbol{\Psi} \tilde{Q}
$$

$$
\tilde{\boldsymbol{Q}}=\boldsymbol{\Psi}^{\mathrm{T}} \tilde{\boldsymbol{f}}
$$

where the $N$ input forces are represented by the power flow excitation vector $\tilde{\boldsymbol{Q}}$. The $j$ th power flow excitation force element $\tilde{Q}_{j}$ corresponds to the $j$ th mobility power flow mode $\psi_{j}$. Similarly, because $\sum_{j=1}^{N}\left|\tilde{Q}_{j}\right|^{2}=\sum_{j=1}^{N}\left|\tilde{f}_{j}\right|^{2}$, the timeaveraged power input of the $N$ forces described in equation (5.1) takes the form

$$
P=\frac{1}{2} \operatorname{Re}\left\{\tilde{\boldsymbol{Q}}^{\mathrm{H}} \boldsymbol{\Xi} \tilde{\boldsymbol{Q}}\right\}=\frac{1}{2} \sum_{i=1}^{N} \mu_{i}\left|\tilde{Q}_{i}\right|^{2},
$$

and the corresponding time-averaged power estimation is given by

$$
P_{\text {low }}=\frac{1}{2}\left(\sum_{j=1}^{N}\left|\tilde{f}_{j}\right|^{2}\right) \mu_{1}<P<\frac{1}{2}\left(\sum_{j=1}^{N}\left|\tilde{f}_{j}\right|^{2}\right) \mu_{N}=P_{\text {up }} .
$$

\section{(b) Comparison of the two power flow mode approaches}

Table 1 provides a comparison of the two power flow mode mathematical models in terms of their inherent characteristics and definitions.

As shown in this table, the differences between the two power flow mode approaches are as follows.

(i) In the damping power flow model (DPFM) the characteristic matrix relates only to the system's damping property, whereas in the mobility power flow model (MPFM) the system's mobility matrix requires full knowledge of damping, stiffness, mass and excitation frequency.

(ii) The characteristic factor $\lambda_{j}$ of DPFM relies only on the system's damping property. If the damping property is independent of the excitation frequency (for example, only viscous damping modelling is used and hysteresis material damping is neglected, i.e. $\eta=0$ ), DPFM is independent of excitation frequency. However, for MPFM, the characteristic factor $\mu_{j}$ needs mobility information involving mass, stiffness, damping and excitation frequency, even if the damping property does not relate to the excitation frequency. 
Table 1. Comparison of the two power flow mode mathematical models

\begin{tabular}{|c|c|c|}
\hline \multirow[t]{2}{*}{ characteristic } & \multicolumn{2}{|l|}{ model } \\
\hline & $\begin{array}{l}\text { damping-characterized power } \\
\text { flow mode model (DPFM) }\end{array}$ & $\begin{array}{l}\text { mobility-characterized power flow } \\
\text { mode model (MPFM) }\end{array}$ \\
\hline \multirow[t]{2}{*}{ characteristic matrix } & $\begin{array}{l}\text { characteristic-damping matrix } \\
\overline{\boldsymbol{C}}=\frac{1}{2}\left(\boldsymbol{C}+\boldsymbol{C}^{\mathrm{T}}\right) \\
\text { depending only on system's } \\
\quad \text { damping }\end{array}$ & $\begin{array}{l}\text { characteristic mobility matrix } \\
\overline{\boldsymbol{Y}}=\frac{1}{2}\left(\tilde{\boldsymbol{Y}}+\tilde{\boldsymbol{Y}}^{\mathrm{H}}\right) \\
\text { depending on system's inertia, } \\
\quad \text { elastic, damping parameters } \\
\text { and exciting frequency }\end{array}$ \\
\hline & $\overline{\boldsymbol{C}}=\boldsymbol{\Phi} \boldsymbol{\Lambda} \boldsymbol{\Phi}^{\mathrm{T}}$ & $\overline{\boldsymbol{Y}}=\boldsymbol{\Psi} \boldsymbol{\Xi} \boldsymbol{\Psi}^{\mathrm{T}}$ \\
\hline \multicolumn{3}{|l|}{ power flow space } \\
\hline \multirow{2}{*}{$\begin{array}{l}\text { power flow mode: eign- } \\
\text { vectors of the charac- } \\
\text { teristic matrix }\end{array}$} & $\boldsymbol{\Phi}=\left[\boldsymbol{\varphi}_{1}, \boldsymbol{\varphi}_{2}, \ldots, \boldsymbol{\varphi}_{N}\right]$ & $\boldsymbol{\Psi}=\left[\psi_{1}, \psi_{2}, \ldots, \psi_{N}\right]$ \\
\hline & $\boldsymbol{\Phi}^{\mathrm{T}} \boldsymbol{\Phi}=\boldsymbol{\Phi} \boldsymbol{\Phi}^{\mathrm{T}}=\boldsymbol{I}$ & $\boldsymbol{\Psi} \boldsymbol{\Psi}^{\mathrm{T}}=\boldsymbol{\Psi}^{\mathrm{T}} \boldsymbol{\Psi}=\boldsymbol{I}$ \\
\hline characteristic factor & $\Lambda=\operatorname{diag}\left[\lambda_{j}\right]$ & $\boldsymbol{\Xi}=\operatorname{diag}\left[\mu_{j}\right]$ \\
\hline units & $\mathrm{Ns} \mathrm{m}^{-1}$ & $\mathrm{~m}(\mathrm{Ns})^{-1}$ \\
\hline power flow variable & response vector $\tilde{\boldsymbol{q}}=\boldsymbol{\Phi}^{\mathrm{T}} \tilde{\boldsymbol{V}}$ & excitation vector $\tilde{\boldsymbol{Q}}=\boldsymbol{\Psi}^{\mathrm{T}} \tilde{\boldsymbol{f}}$ \\
\hline power flow formulation & $P=\frac{1}{2} \operatorname{Re}\left\{\tilde{\boldsymbol{q}}^{\mathrm{H}} \boldsymbol{\Lambda} \tilde{\boldsymbol{q}}\right\}=\frac{1}{2} \sum_{j=1}^{N} \lambda_{j}\left|\tilde{q}_{j}\right|^{2}$ & $P=\frac{1}{2} \operatorname{Re}\left\{\tilde{\boldsymbol{Q}}^{\mathrm{H}} \boldsymbol{\Xi} \tilde{\boldsymbol{Q}}\right\}=\frac{1}{2} \sum_{j=1}^{N} \mu_{j}\left|\tilde{Q}_{j}\right|^{2}$ \\
\hline$j$ th mode dissipation power & $P_{j}=\lambda_{j}\left|\tilde{q}_{j}\right|^{2} / 2$ & $P_{j}=\mu_{j}\left|\tilde{Q}_{j}\right|^{2} / 2$ \\
\hline physical meaning & $\begin{array}{l}\lambda_{j} \text { represents the energy dissipated } \\
\quad \text { per unit power flow response } \tilde{q}_{j}\end{array}$ & $\begin{array}{l}\mu_{j} \text { represents the energy dissi- } \\
\text { pated per unit power flow } \\
\text { excitation } \tilde{Q}_{j}\end{array}$ \\
\hline power flow bounds & $P_{\text {low }}=\frac{1}{2}\left(\sum_{j=1}^{N}\left|\tilde{v}_{j}\right|^{2}\right) \lambda_{1}$ & $P_{\mathrm{low}}=\frac{1}{2}\left(\sum_{j=1}^{N}\left|\tilde{f}_{j}\right|^{2}\right) \mu_{1}$ \\
\hline & $P_{\mathrm{up}}=\frac{1}{2}\left(\sum_{j=1}^{N}\left|\tilde{v}_{j}\right|^{2}\right) \lambda_{N}$ & $P_{\mathrm{up}}=\frac{1}{2}\left(\sum_{j=1}^{N}\left|\tilde{f}_{j}\right|^{2}\right) \mu_{N}$ \\
\hline & $\begin{array}{l}P_{\text {low }}<P<P_{\text {up }} \\
\frac{\lambda_{1}}{2}<\frac{P}{|\tilde{\boldsymbol{V}}|^{2}}<\frac{\lambda_{N}}{2}\end{array}$ & $\begin{array}{l}P_{\text {low }}<P<P_{\text {up }} \\
\frac{\mu_{1}}{2}<\frac{P}{|\tilde{\boldsymbol{f}}|^{2}}<\frac{\mu_{N}}{2}\end{array}$ \\
\hline
\end{tabular}

(iii) The power flow mode vectors $\boldsymbol{\Phi}$ of DPFM and $\boldsymbol{\Psi}$ of MPFM exhibit the same characteristics described in (ii). Therefore, the power flow space constructed using $\boldsymbol{\Psi}$ changes with the excitation frequency. On the contrary, the power flow space spanned by $\boldsymbol{\Phi}$ is characterized only by the system's damping.

(iv) The total power dissipation of the system is expressed by $P=\sum_{j=1}^{N} \lambda_{j}\left|\tilde{q}_{j}\right|^{2} / 2$ in DPFM and $P=\sum_{j=1}^{N} \mu_{j}\left|\tilde{Q}_{j}\right|^{2} / 2$ in MPFM, respectively. The $j$ th characteristic velocity $\tilde{q}_{j}$ involves the damping property and velocity response of the system, whereas $\tilde{Q}_{j}$ relates to the mobility and the excitation forces. If the velocity is given (for example, prescribed designed 


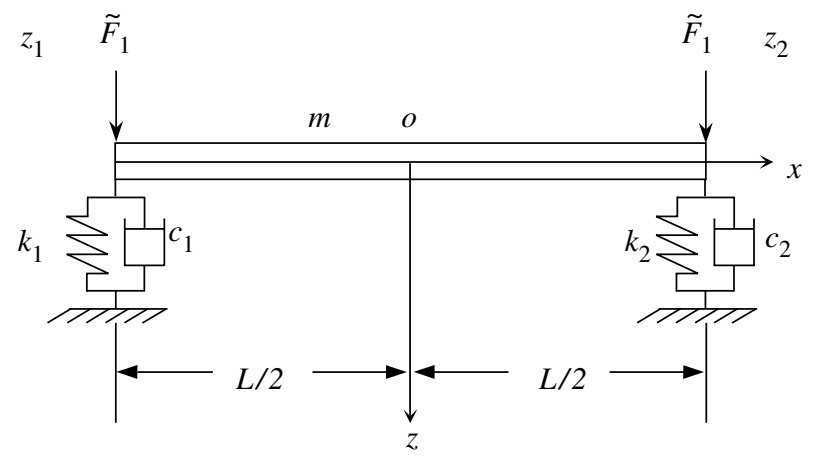

Figure 1. A simple two degrees of freedom system under forced excitation.

motions or experimentally measured), the total dissipated power depends only on the damping characteristics of the system. However, in MPFM, if the excitation forces are known, the total power dissipated again depends only on mobility data involving mass, stiffness, damping and excitation frequency.

(v) In applications presented in $\$ 6$, it is demonstrated that DPFM is convenient for use in power flow design and control because of its dependence on only the damping distribution which can be adjusted as required. In contrast, MPFM requires mobility information which is dependent on frequency and therefore its characteristic factors $\mu_{j}$ and the corresponding power flow mode vectors $\psi_{j}$ vary with frequency. As an explanatory example, we consider a simple idealized linear system with two degrees of freedom under force excitation as shown in figure 1. This system comprises of two mounts, each having viscous damping $c_{1}=50 \mathrm{Ns} \mathrm{m}^{-1}$, $c_{2}=10 \mathrm{Ns} \mathrm{m}^{-1}$ and stiffness $k_{1}=1200 \mathrm{~N} \mathrm{~m}^{-1}, k_{2}=600 \mathrm{~N} \mathrm{~m}^{-1}$, supporting an uniform rigid bar of length $L=1 \mathrm{~m}$ and mass density $\underset{\sim}{\sim}=100 \mathrm{~kg} \mathrm{~m}^{-1}$. The two external sinusoidal excitation forces $\tilde{F}_{j}(t)=\tilde{f}_{j} \mathrm{e}^{\mathrm{i} \omega t}(j=1,2)$, of amplitudes $\tilde{f}_{1}=10, \tilde{f}_{2}=5 \mathrm{e}^{\mathrm{i} \pi / 6}$ and frequency $\omega$ are applied to the ends of the bar.

Numerical results derived by the MPFM of this system are presented in figure 2 which displays the variation of power flow mode forms with frequency as shown in figure $2 a$. Here, the +1 and -1 scale values represent a heave and pitch motion, respectively. Figure $2 b$ illustrates the variation of the characteristic factor $\mu_{j}(j=1,2)$ with frequency. It is observed that at frequencies $f_{1}, f_{2}$ and $f_{3}$ shown in $(a)$ the forms of the power flow mode associated with $\mu_{1}(---)$ and $\mu_{2}$ $(\cdots)$ are exchanged. This causes a jump phenomena in the values of the characteristic factor $\mu_{j}(j=1,2)$ in $(b)$ and the power flow dissipations $P_{\mathrm{m} 1}$ and $P_{\mathrm{m} 2}$ (shown in $(c)$ ) corresponding to each mode. For the same system, numerical results predicted by the DPFM are presented in $\$ 6 a$, where the characteristic factors $\lambda_{1}=10, \lambda_{2}=50$ are constants and hence there are no such jumps in the mode power characteristics as shown in figure 3. For a complex system including material hysteresis damping $(\eta \neq 0)$ which causes a frequency dependent characteristic-damping matrix $\overline{\boldsymbol{C}}$, a further comparison is presented in $\S 6 c$ with associated figures 14 and 15 for illustrative purposes. 

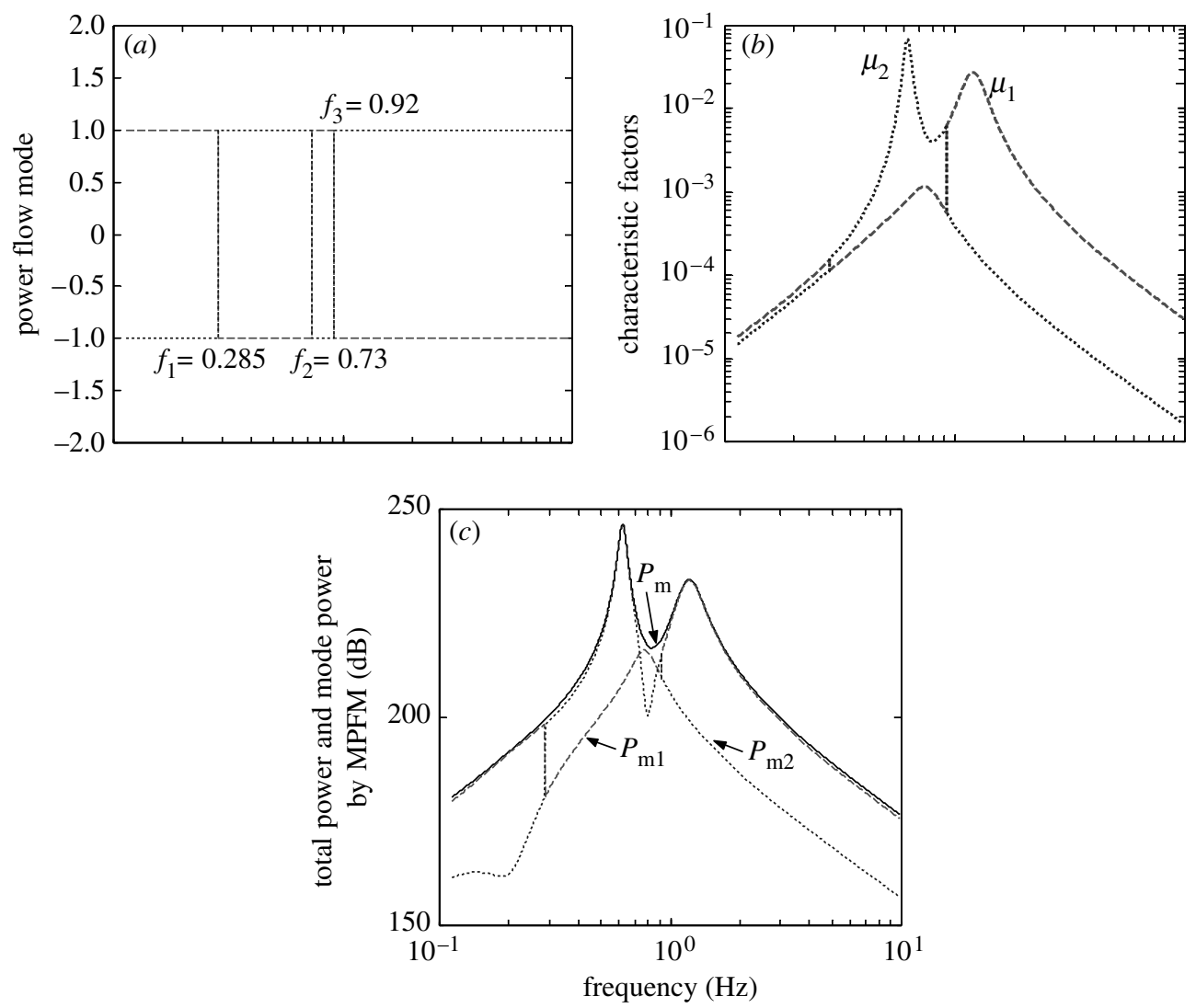

Figure 2. Numerical results derived by the mobility based power flow mode method for the system shown in figure 1 and illustrate variation of responses with frequency: $(a)$ power flow mode forms; $(b)$ characteristic factors; $(c)$ power flow dissipations. (--- denotes the 1 st mode, $\cdots$ the 2 nd mode result).

\section{Applications of damping power flow mathematical model}

\section{(a) A force excited system}

Let us examine the dynamic behaviour of the system shown in figure 1 using the damping based power flow mode theory. The equation describing the dynamics of this system can be expressed in the matrix form

$$
\left[\begin{array}{cc}
m L / 3 & m L / 6 \\
m L / 6 & m L / 3
\end{array}\right]\left\{\begin{array}{l}
\ddot{z}_{1} \\
\ddot{z}_{2}
\end{array}\right\}+\left[\begin{array}{cc}
c_{1} & 0 \\
0 & c_{2}
\end{array}\right]\left\{\begin{array}{l}
\dot{z}_{1} \\
\dot{z}_{2}
\end{array}\right\}+\left[\begin{array}{cc}
k_{1} & 0 \\
0 & k_{2}
\end{array}\right]\left\{\begin{array}{l}
z_{1} \\
z_{2}
\end{array}\right\}=\left\{\begin{array}{l}
\tilde{F}_{1} \\
\tilde{F}_{2}
\end{array}\right\},
$$

where the damping matrix $\boldsymbol{C}$ is real and symmetric, thus $\overline{\boldsymbol{C}}=\boldsymbol{C}$. To predict the system's input power flow using the proposed damping-based power flow mode theory, the eigenvalues $\lambda_{j}$ of the characteristic-damping matrix $\bar{C}$ are of values $\lambda_{1}=10 \mathrm{Ns} \mathrm{m}^{-1}, \lambda_{2}=50 \mathrm{Ns} \mathrm{m}^{-1}$ with $j=1,2$.

The time-averaged total input power flow $P=P_{1}+P_{2}$ and the mode dissipation power are calculated using equations (3.11) and (3.12), respectively. Figure 3 displays the time-averaged input power $P$ and two mode dissipation powers $P_{1}$ and $P_{2}$. As demonstrated by calculations, the total input power flow $P$ 


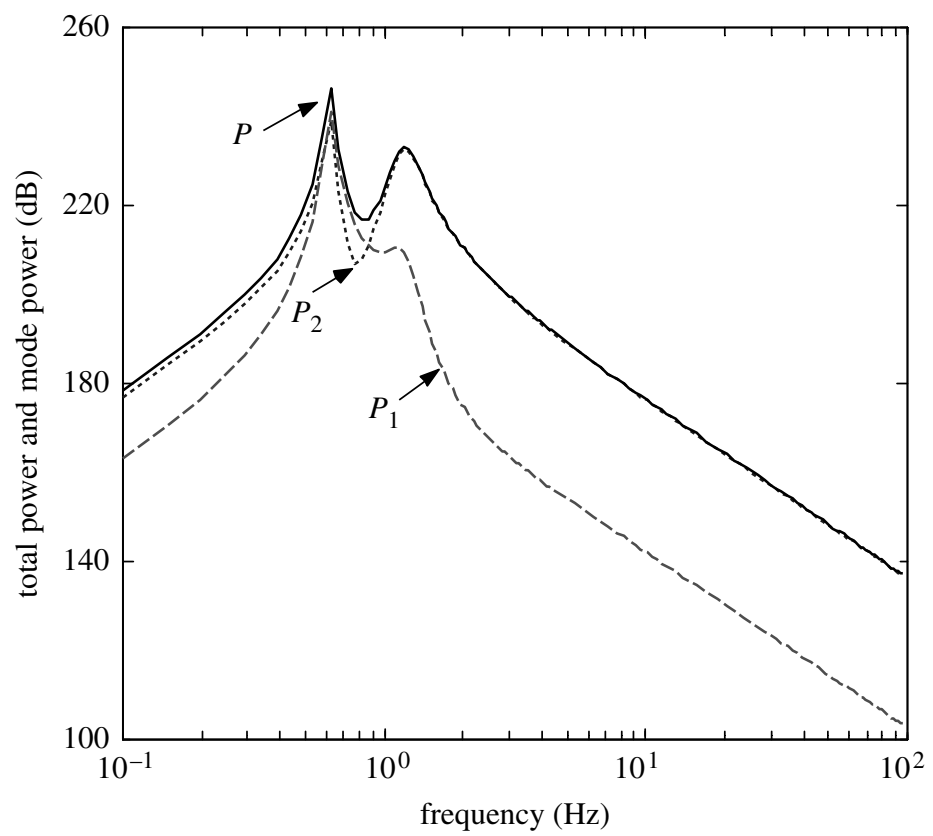

Figure 3. Total input power $P$, the first mode dissipation power $P_{1}$ and the second mode dissipation power $P_{2}\left(c_{1}=50 \mathrm{Ns} \mathrm{m}^{-1}, c_{2}=10 \mathrm{Ns} \mathrm{m}^{-1}\right)$.

equals the value determined using the traditional method of Goyder \& White (1980) by multiplying the force and velocity responses given in equation (3.1).

In this example, the damping matrix is diagonal and its two characteristicdamping factors equal its two physical damping coefficients, that is $\lambda_{1}=10=c_{2}$ and $\lambda_{2}=50=c_{1}$, and the power flow mode vector matrix is a unit matrix. As shown in equation (3.12), the mode dissipation power $P_{j}=\lambda_{j}\left|\tilde{q}_{j}\right|^{2} / 2(j=1,2)$ for the $j$ th power flow mode depends on both the characteristic-damping factor $\lambda_{j}$ and the modulus of the power flow response $\tilde{q}_{j}$. Therefore, in a range of frequencies near to the dip in the curve, the mode dissipation power $P_{1}$ is larger than $P_{2}$, although the characteristic-damping factor $\lambda_{1}$ is less than $\lambda_{2}$.

Figure 4 shows estimations of the total input power, its upper and lower bounds and illustrates how the total power varies with frequency between these outer limits.

Figure 5 shows the special case of $c_{1}=c_{2}=10=c$, for which the eigenvalues of the characteristic-damping matrix are the same, i.e. $\lambda_{1}=\lambda_{2}=c$. Therefore, $P_{\text {low }}=$ $P=P_{\text {up }}$ and the three curves overlap as shown. However, the two mode dissipation powers $P_{1}$ and $P_{2}$ are different because the power flow responses $\tilde{q}_{j}$ $(j=1,2)$ are different.

Figure 6 illustrates the effect of the damping ratio $a$ on the total input power spectra. It is observed that the peaks of the total power reduce in value and the power flow spectra become flatter as the damping ratio increases. This is dramatically observed when damping ratio $a=50$ causing the second power flow peak in the spectrum to decrease in value significantly.

Figure 7 shows the behaviour of the time-averaged input power $P$ of a symmetric suspension system $\left(k_{1}=1200 \mathrm{~N} \mathrm{~m}^{-1}=k_{2}, c_{1}=c=c_{2}, \tilde{f}_{1}=10 \mathrm{~N}=\tilde{f}_{2}\right)$ influenced by 


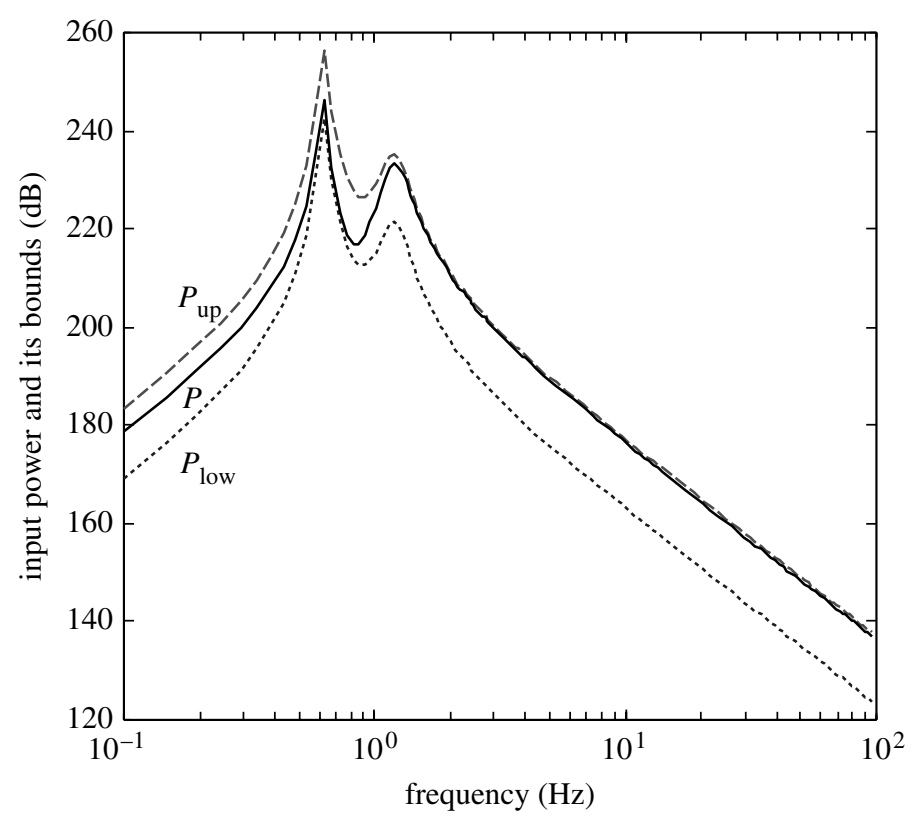

Figure 4. Total power with its upper and lower bounds.

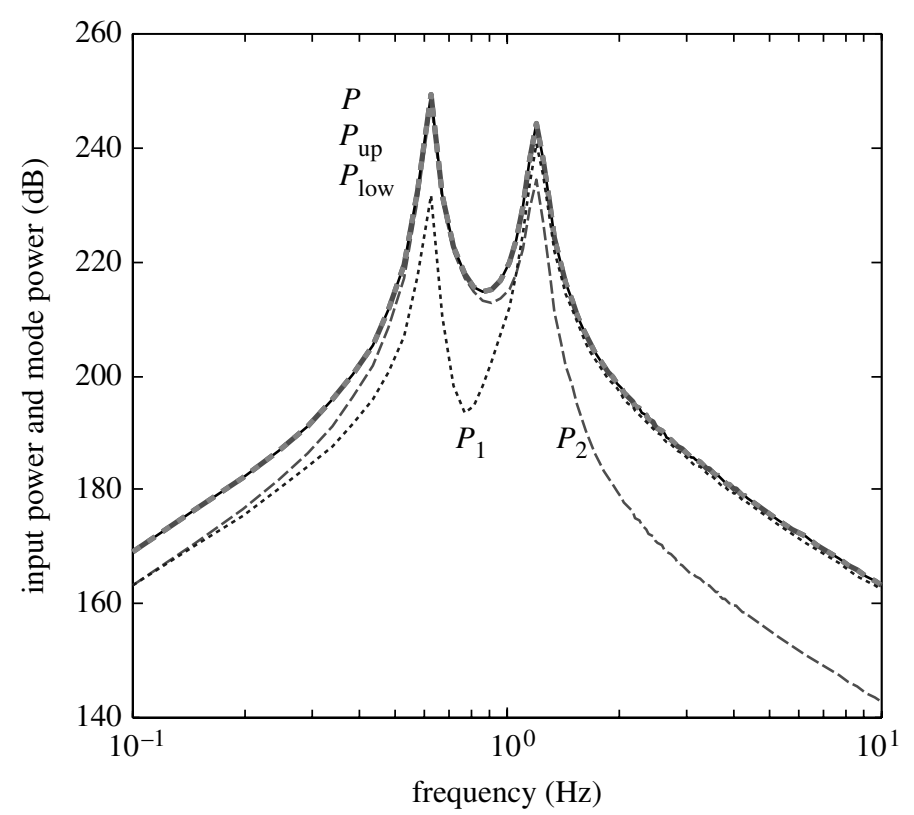

Figure 5. Total power with its upper and lower bounds and mode dissipation powers when $c_{1}=$ $c_{2}=10 \mathrm{Ns} \mathrm{m}^{-1}$.

varying damping values. Again, in this case, the total and individual mode dissipation powers are the same, i.e. $P_{\text {low }}=P=P_{\text {up }}$. Because only heave motion is excited in this system, only one peak exists in the power spectra. We again observe that as the values of damping $c_{1}=c_{2}$ increase the curves flatten. 


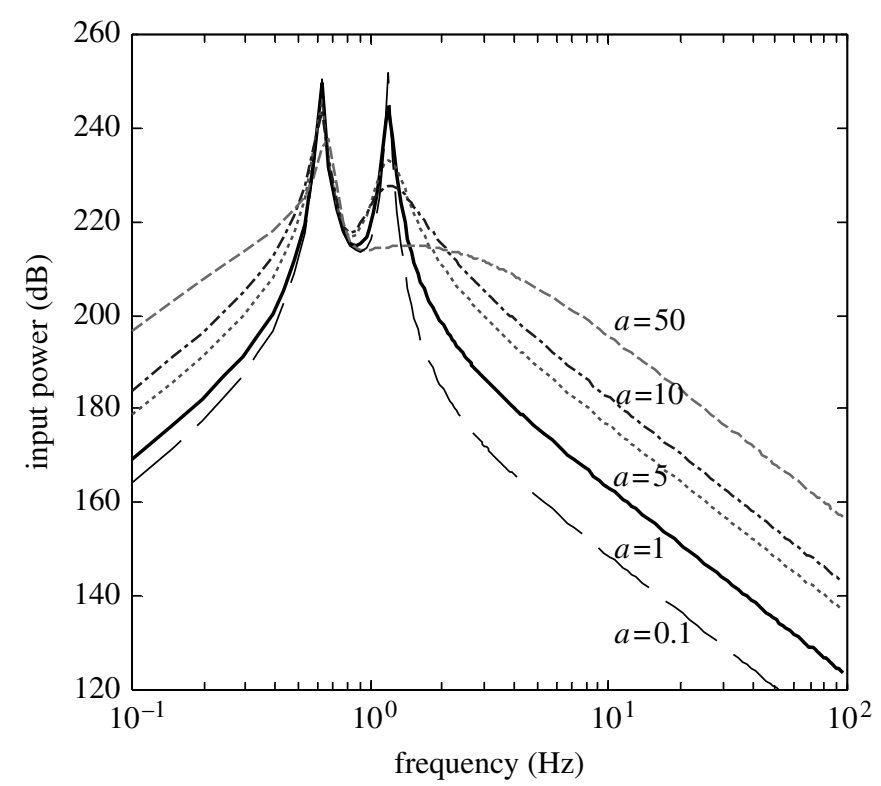

Figure 6. Total input power influenced by the system's damping properties $\left(a=c_{1} / c_{2}, c_{2}=10 \mathrm{Ns} \mathrm{m}^{-1}\right)$.

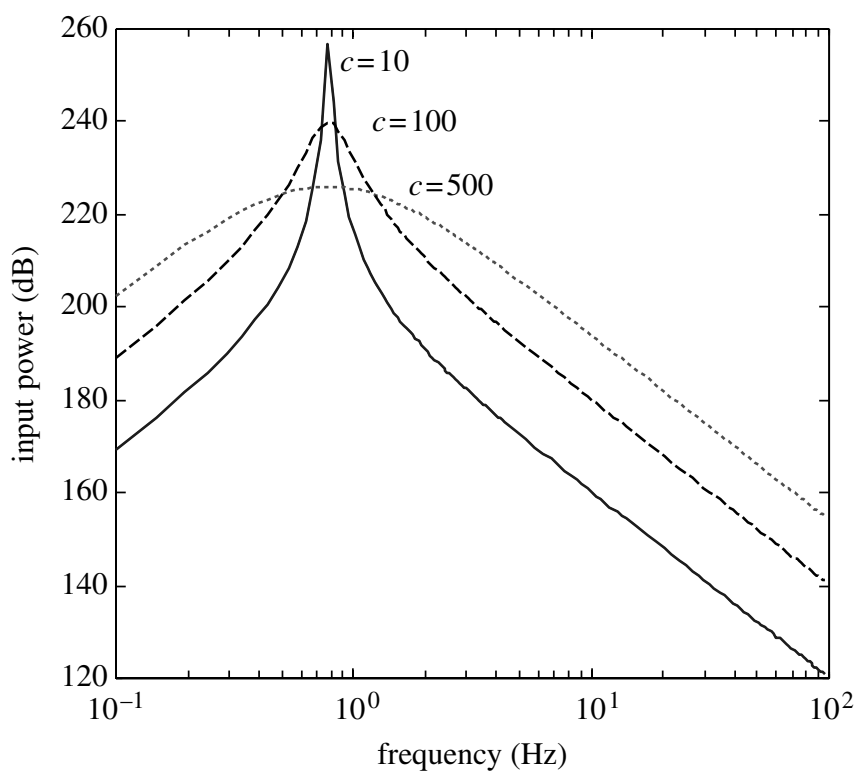

Figure 7. Total input power in overall symmetric system affected by damping properties.

(b) A simple vehicle suspension system subject to motion excitations

Figure 8 illustrates a simple vehicle suspension system to model different components of the design (Rahnejat 1998). In this example, the system is subject to road disturbance, i.e. the vibration source is a motion rather than a forced excitation. To predict the input power, the power flow mode approach is applied without a full knowledge of the force excitation. 


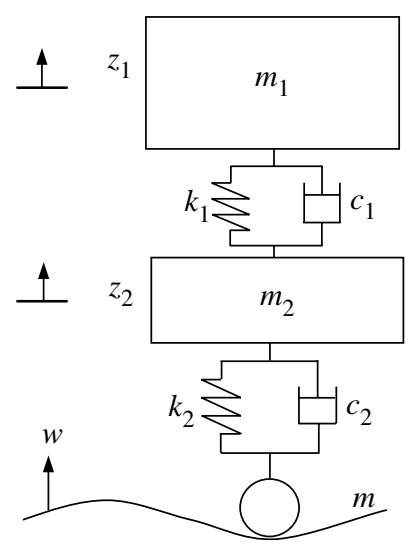

Figure 8. A simple wheel car suspension system.

The parameters chosen in this example are: main body mass $m_{1}=2500 \mathrm{~kg}$, suspension mass $m_{2}=320 \mathrm{~kg}$, wheel and tyre mass $m=25 \mathrm{~kg}$, spring constant of suspension system $k_{1}=80000 \mathrm{~N} \mathrm{~m}^{-1}$, spring constant of wheel and tyre $k_{2}=500000 \mathrm{~N} \mathrm{~m}^{-1}$, damping constant of suspension system $c_{1}=350 \mathrm{Ns} \mathrm{m}^{-1}$, damping constant of wheel and tyre $c_{2}=15020 \mathrm{Ns} \mathrm{m}^{-1}$. It is assumed that the representative road disturbance is described by a road profile $w=W \mathrm{e}^{\mathrm{j} \omega t}$. The equation describing the dynamics of this suspension system is given by

$$
\begin{aligned}
& {\left[\begin{array}{ccc}
m_{1} & 0 & 0 \\
0 & m_{2} & 0 \\
0 & 0 & m
\end{array}\right]\left\{\begin{array}{c}
\ddot{z}_{1} \\
\ddot{z}_{2} \\
\ddot{w}
\end{array}\right\}+\left[\begin{array}{ccc}
c_{1} & -c_{1} & 0 \\
-c_{1} & c_{1}+c_{2} & -c_{2} \\
0 & -c_{2} & c_{2}
\end{array}\right]\left\{\begin{array}{c}
\dot{z}_{1} \\
\dot{z}_{2} \\
\dot{w}
\end{array}\right\} } \\
&+ {\left[\begin{array}{ccc}
k_{1} & -k_{1} & 0 \\
-k_{1} & k_{1}+k_{2} & -k_{2} \\
0 & -k_{2} & k_{2}
\end{array}\right]\left\{\begin{array}{c}
z_{1} \\
z_{2} \\
w
\end{array}\right\}=\left\{\begin{array}{l}
0 \\
0 \\
F
\end{array}\right\}, }
\end{aligned}
$$

where $F(t)$ is an unknown interaction force between the tyre and the road surface.

The system's damping matrix $\boldsymbol{C}$ is real and symmetric (i.e. $\overline{\boldsymbol{C}}=\boldsymbol{C}$ ) and the eigenvalues of the characteristic-damping matrix $\bar{C}$ are determined as $\lambda_{1}=0$, $\lambda_{2}=522$ and $\lambda_{3}=30218$.

Figure 9 displays the total time averaged input power $P$ compared to calculations derived using the traditional power flow method (Goyder \& White 1980), together with the energy dissipated by non-zero eigenvalue power flow modes $P_{2}$ and $P_{3}$. An application of the proposed theory gives $P_{1}=0$ since $\lambda_{1}=0$ (see equation (3.12)), which implies that the energy dissipated by the first power flow mode is zero. In fact, $\lambda_{1}=0$ corresponds to a rigid vibration mode, hence the damper does not dissipate any vibratory energy. $P_{1}=0$ also indicates the lower bound of the input power which is not included in the figure. The total power predicted by the dampingcharacterized power flow mode and the mobility-based power flow mode mathematical models produce equivalent results. However, the latter requires more computing time because the mobility expression is dependent on frequency, hence, eigenvalues are solved for each frequency step as described in $\$ 5 b$. In contrast, the damping matrix $\boldsymbol{C}$ (as chosen) is independent of frequency and, 


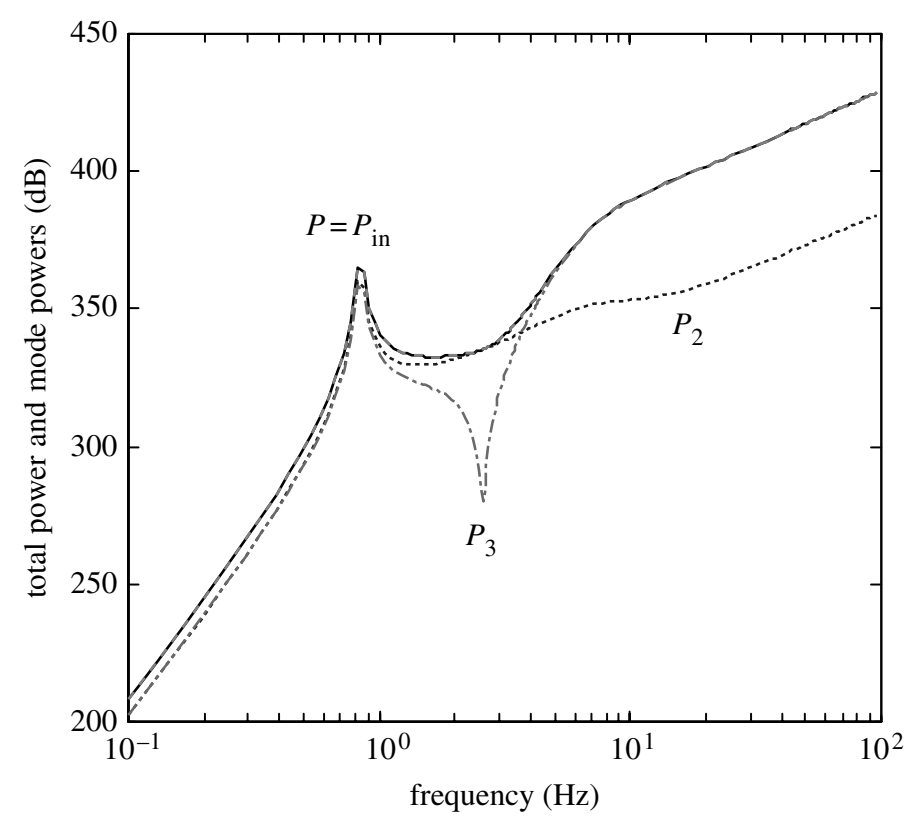

Figure 9. Total input power $P$ to the system and non-zero power flow modes.

therefore, its eigenvalues are solved only once outside the frequency loop. This demonstrates benefit of application over the mobility-based power flow mode method. Moreover, the total time-averaged power calculated by the dampingcharacterized power flow mode method equals to the predicted value obtained by the traditional method (Goyder \& White 1980), i.e. $P=P_{\text {in }}$.

Figure 10 shows the total time averaged input power $P$ and non-zero power flow modes $P_{2}$ and $P_{3}$ for the suspension system but with damping: $c_{1}=$ $c_{2}=1 \mathrm{Ns} \mathrm{m}^{-1}$ and $k_{1}, k_{2}$ unchanged. In this figure, the top dashed line represents the total input power of the original system $\left(c_{1}=350 \mathrm{Ns} \mathrm{m}^{-1}, c_{2}=15020 \mathrm{Ns} \mathrm{m}^{-1}\right)$. It is seen from figure 10 that the peaks of the total time-averaged power $P$ for small damping are more pronounced than the case of large damping (the thin line). However, the level of the total time-averaged power reduces as the damping reduces. This suggests that a system with small damping component values has less capacity for energy dissipation. Consequently, the value of the input power is small.

\section{(c) Active control system with a non-symmetric damping matrix}

The third example analyses a system incorporating beam material damping and active controls producing a non-symmetric damping matrix. Figure 11 provides a schematic illustration of this system used to model the interactions between a human body, seat, suspension system and flexible vessel experiencing wave excitations (Xiong et al. 2003b). To improve ride comfort, two active controllers producing active damping are incorporated into the system. The damping-based power flow mode method is again applied to investigate this discrete-compliant system under wave excitation.

The equation describing the system's dynamic interactions is expressed in the form

$$
\boldsymbol{M} \ddot{\boldsymbol{Z}}(t)+\boldsymbol{C} \dot{\boldsymbol{Z}}(t)+\boldsymbol{K} \boldsymbol{Z}(t)=\boldsymbol{F} .
$$




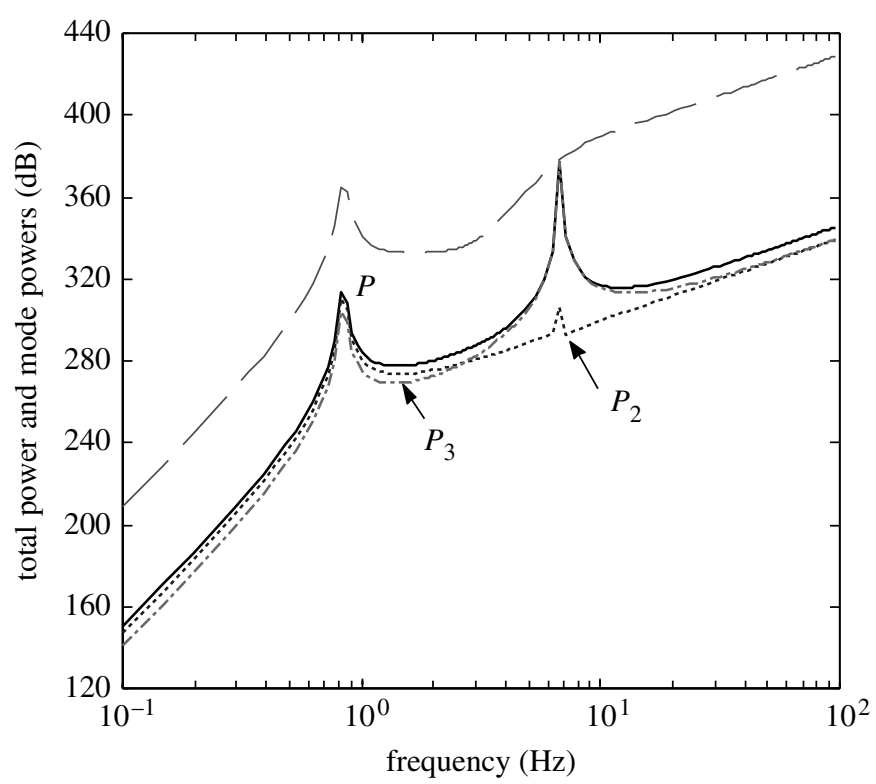

Figure 10. Total input power $P$ and non-zero mode dissipation powers $P_{2}$ and $P_{3}$ in a suspension system with small damping $c_{1}=c_{2}=1 \mathrm{Ns} \mathrm{m}^{-1}$ compared to original system illustrated in figure 9 (i.e. $c_{1}=350 \mathrm{Ns} \mathrm{m}^{-1}, c_{2}=15020 \mathrm{Ns} \mathrm{m}^{-1}$, top dashed line).

Detailed information of the matrices $\boldsymbol{M}, \boldsymbol{C}$ and $\boldsymbol{K}$ was discussed by Xiong et al. (2003b). In this analysis, however, the damping matrix $\boldsymbol{C}$, defined in equation (2.1b), has the following components:

$$
\boldsymbol{C}_{\mathrm{v}}=\left[\begin{array}{cccccc}
c_{\mathrm{h}} & -c_{\mathrm{h}} & 0 & 0 & \ldots & 0 \\
-c_{\mathrm{h}} & c_{\mathrm{h}}+g_{\mathrm{sp}} & -g_{\mathrm{bp}} W_{0 \mathrm{c}} & -g_{\mathrm{bp}} W_{1 \mathrm{c}} & \ldots & -g_{\mathrm{bp}} W_{n \mathrm{c}} \\
0 & -g_{\mathrm{sp}} W_{0 \mathrm{c}} & C_{0}+g_{\mathrm{bp}} W_{0 \mathrm{c}}^{2} & g_{\mathrm{bp}} W_{0 \mathrm{c}} W_{1 \mathrm{c}} & \ldots & g_{\mathrm{bp}} W_{0 \mathrm{c}} W_{n \mathrm{c}} \\
0 & -g_{\mathrm{sp}} W_{1 \mathrm{c}} & g_{\mathrm{bp}} W_{0 \mathrm{c}} W_{1 \mathrm{c}} & C_{1}+g_{\mathrm{bp}} W_{1 \mathrm{c}}^{2} & \ldots & g_{\mathrm{bp}} W_{1 \mathrm{c}} W_{n \mathrm{c}} \\
\vdots & \vdots & \vdots & \vdots & \ddots & \vdots \\
0 & -g_{\mathrm{sp}} W_{n \mathrm{c}} & g_{\mathrm{bp}} W_{0 \mathrm{c}} W_{n \mathrm{c}} & g_{\mathrm{bp}} W_{1 \mathrm{c}} W_{n \mathrm{c}} & \ldots & C_{n}+g_{\mathrm{bp}} W_{n \mathrm{c}}^{2}
\end{array}\right],
$$

where $g_{\mathrm{sp}}=c_{\mathrm{s}}+g_{\mathrm{s}}, g_{\mathrm{bp}}=c_{\mathrm{s}}+g_{\mathrm{b}}, g_{\mathrm{s}}$ and $g_{\mathrm{b}}$ represent the two control gains shown in figure 10. The parameters $W_{j c}, C_{j}$ and $C_{\mathrm{h} j}(j=0,1,2, \ldots, n)$, represent $n+1$ ship modes, viscous fluid damping and the material damping of the ship, respectively. In this active control system, the feedback gains $g_{\mathrm{s}}$ and $g_{\mathrm{b}}$ can be chosen independently and it is assumed that $g_{\mathrm{sp}} \neq g_{\mathrm{bp}}$ causing both damping and mobility matrices $\boldsymbol{C}$ and $\tilde{\boldsymbol{Y}}$ to be non-symmetric. Therefore, the mobility power flow mode method proposed by Ji et al. (2003) is not applicable. However, the characteristic-damping matrix $\overline{\boldsymbol{C}}$ and characteristic mobility $\overline{\boldsymbol{Y}}$ (defined in equation (5.2)) remain symmetric and hence the power flow mode theories discussed herein are applicable. 


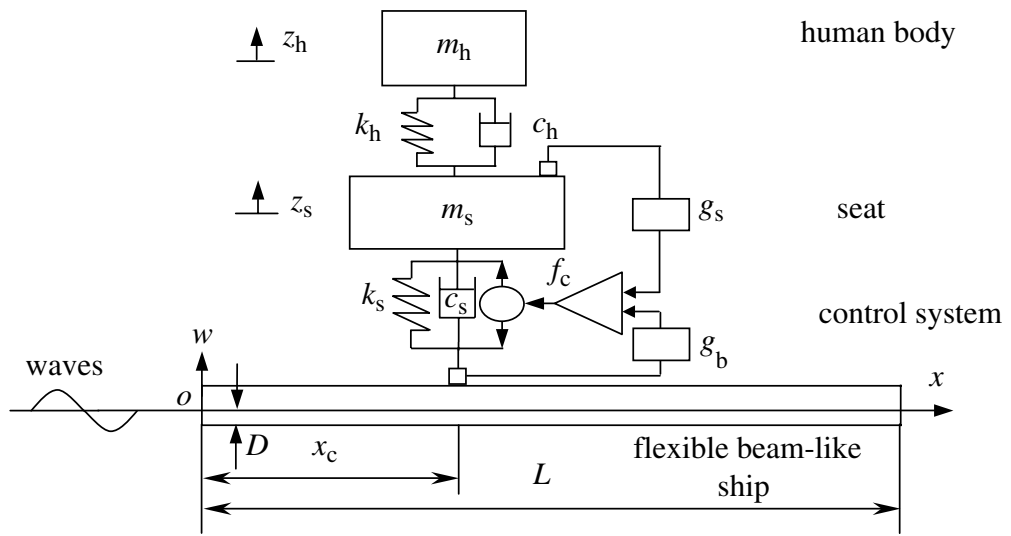

Figure 11. Dynamic model of vessel-seat-human body interactive system with passive and active feedback controls.

For simplification of numerical simulations in a hydroelasticity analysis (Bishop \& Price 1979) of the beam-like ship, three beam modes are chosen $(n=0,1,2)$ together with a two-degrees-of-freedom human body and seat suspension subsystem. Hence the matrix $C$ is of order five $(N=5)$. For illustrative purposes, a sinusoidal wave of wavelength $30 \mathrm{~m}$ and wave amplitude $a=1 \mathrm{~m}$ are assumed. The parameters of the human body and seat suspension subsystem are assumed to have values: $m_{\mathrm{h}}=37.3 \mathrm{~kg}, \quad k_{\mathrm{h}}=36667 \mathrm{~N} \mathrm{~m}^{-1}, \quad c_{\mathrm{h}}=450 \mathrm{Ns} \mathrm{m}^{-1}, \quad m_{\mathrm{s}}=59.7 \mathrm{~kg}, \quad k_{\mathrm{s}}=$ $14350 \mathrm{~N} \mathrm{~m}^{-1}$ and $c_{\mathrm{s}}=600 \mathrm{Ns} \mathrm{m}^{-1}$. The vessel is modelled as a composite uniform beam of length $L=9 \mathrm{~m}$, width $B=2.31 \mathrm{~m}$, draught $D=0.235 \mathrm{~m}$, second moment of area about the neutral axis $I=0.0768 \mathrm{~m}^{4}$, mass per unit length $\rho_{\mathrm{b}}=555.6 \mathrm{~kg} \mathrm{~m}^{-1}$, Young's modulus of the composite beam $E=25.91 \mathrm{Gpa}$ and a range of loss factor values, i.e. $\eta=0,0.1,0.01,0.5$, considered to assess sensitivity of predicted values. The seat is located at $3.94 \mathrm{~m}$ from the stern of the boat, i.e. $x_{\mathrm{c}}=0.438 \mathrm{~L}$, corresponding to a position approximately in the middle of the cockpit area of the real vessel. The position of the seat can be readily changed to analyse the motions for different seating arrangements reflecting number of crewmembers.

Figure 12 shows the time-averaged total input power $P$ and each mode dissipation power $P_{j}(1 \leq j \leq 5)$ assuming a passive control system with no material damping $(\eta=0)$. In this case, the characteristic-damping matrix $\overline{\boldsymbol{C}}$ is independent of frequency. Hence the eigenvalues of $\overline{\boldsymbol{C}}$ are of values $\lambda=124.1$, $914.3,1062.6,2744.7$ and 3280.3 and independent of frequency.

When considering the influence of hysteresis damping of the material in the beam-like ship (i.e. $\eta=0.1,0.5$ ), the characteristic-damping matrix $\overline{\boldsymbol{C}}$ in equation (3.7) is frequency dependent. Therefore, the eigenvalues of matrix $\overline{\boldsymbol{C}}$ are frequency dependent and need solving at each frequency. Figure 13 displays the total input power $P$ of the passive control system influenced by material damping in the beam-like ship. These findings demonstrate that the power dissipation curves with large values of hull damping are flatter producing the corresponding large areas between the curves and the frequency axis, which implies that a large value of hull damping globally increases the power dissipated.

To compare numerical results involving material damping $(\eta \neq 0)$ for component powers decomposed in the two different power flow mode approaches, figure 14a,b 


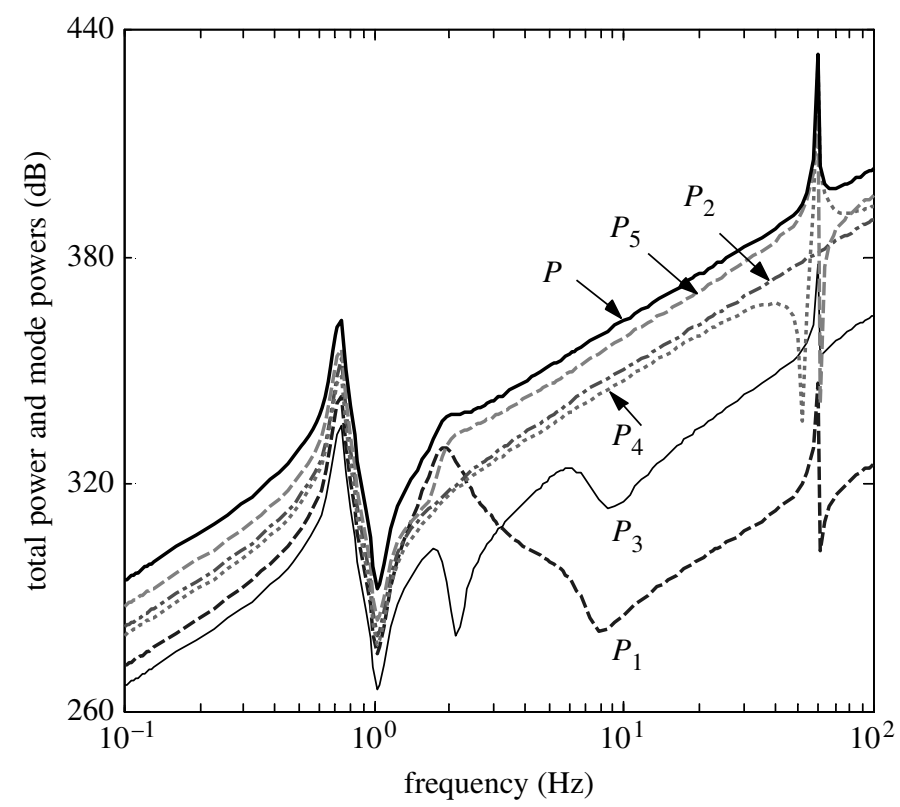

Figure 12. Total power and each mode dissipation power of the passive system with no material damping $(\eta=0)$.

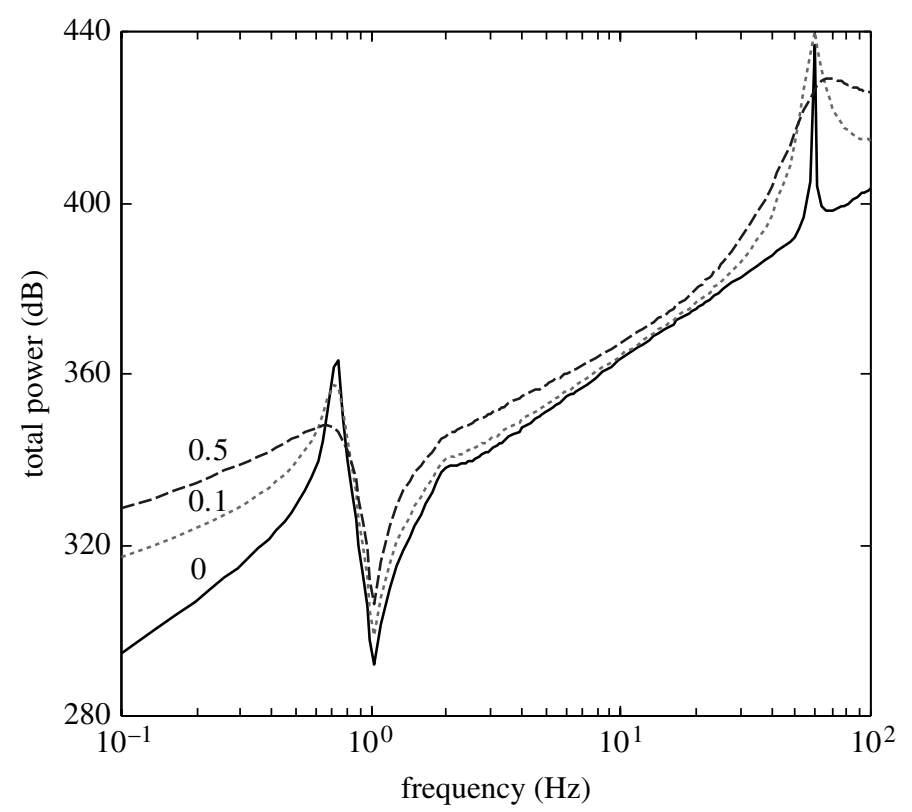

Figure 13. Total input power of the passive control system influenced by the material damping of the beam-like ship in the coupled system: wave length $=30 \mathrm{~m}, \eta=0,0.1,0.5$.

present the power dissipation component $P_{j}$ by each power mode using the DPFM and $P_{\mathrm{m} j}$ by mobility MPFM methods, respectively, whereas figure 15 displays the corresponding characteristic factors of the two approaches. The essential difference between each pair of mode powers $P_{j}$ and $P_{\mathrm{m} j}(j=1, \ldots, 5)$ is the jump phenomena 

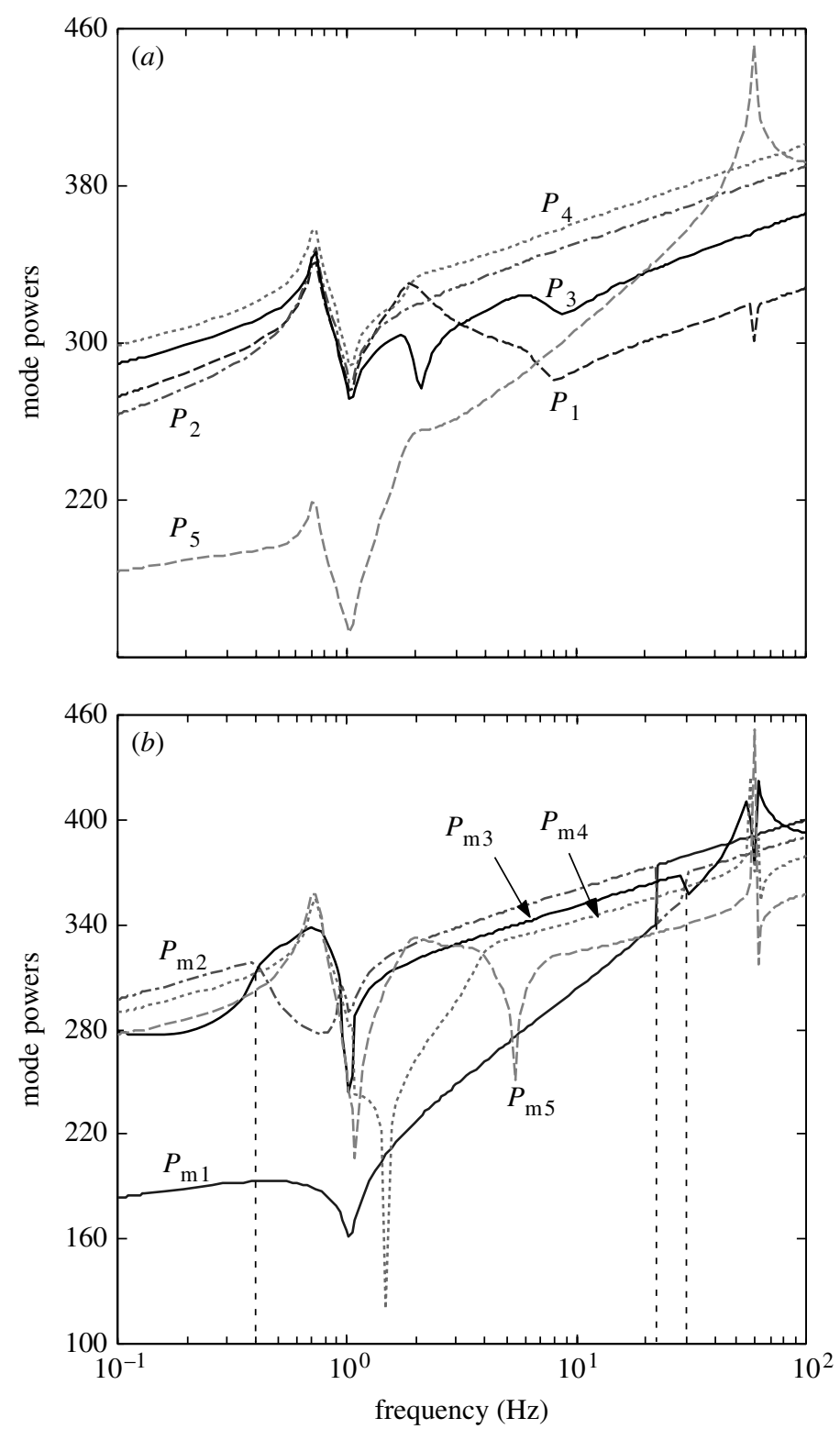

Figure 14. Energy dissipated by each power mode considering material damping of the beam-like ship (wave length $=30 \mathrm{~m}, \eta=0.01$ ) $(a)$ by the damping power flow modes and $(b)$ by the mobility power flow model methods.

occurring at frequencies $0.4,22$ and $30 \mathrm{~Hz}$ in the mode power curves derived by the mobility power flow mode method (see also the comparison between $P_{\mathrm{m} 1}$ and $P_{\mathrm{m} 2}$; $P_{\mathrm{m} 2}$ and $\left.P_{\mathrm{m} 3}\right)$. These jumps are associated with the jump phenomena occurring in the characteristic factor $\mu_{j}$ as shown in figure $15 b$. However, the characteristic factor $\lambda_{j}$ in figure $15 a$ shows a clear pattern for each mode although $\eta=0.01$ makes the characteristic-damping matrix frequency dependent.

Figure 16 illustrates the influence of active control on the power per unit square of velocity vector modulus $P /|\tilde{\boldsymbol{V}}|^{2}$. These findings are compared with a passive 

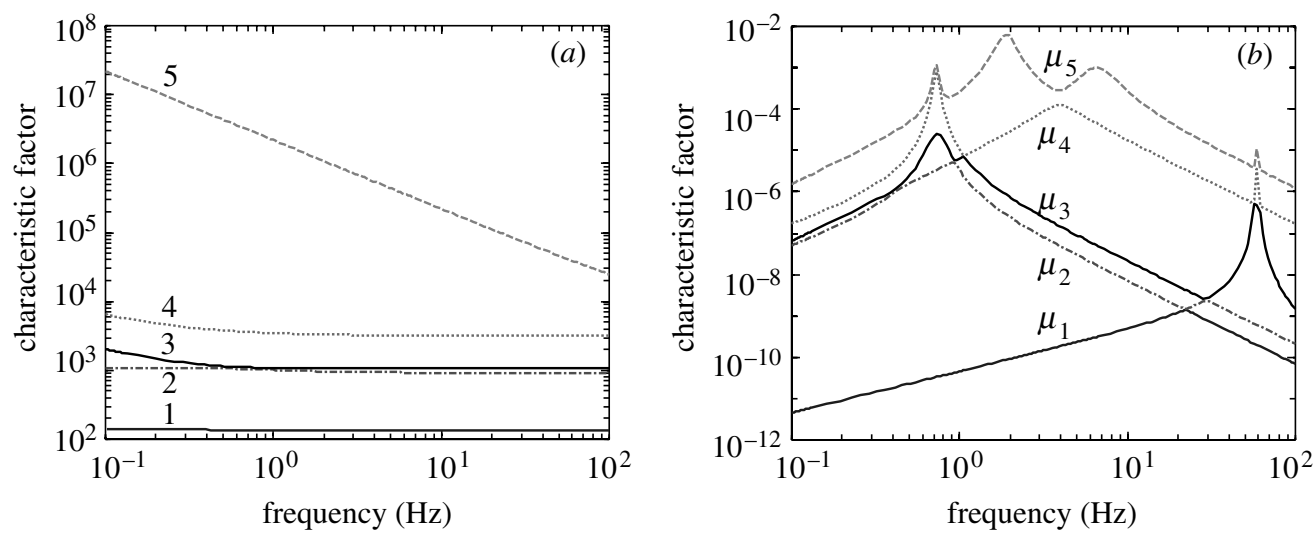

Figure 15. Comparison of the five characteristic factors of the coupled system: $(a)$ by the damping power flow model and $(b)$ by the mobility power flow model methods (wave length $=30 \mathrm{~m}, \eta=0.01$ ).

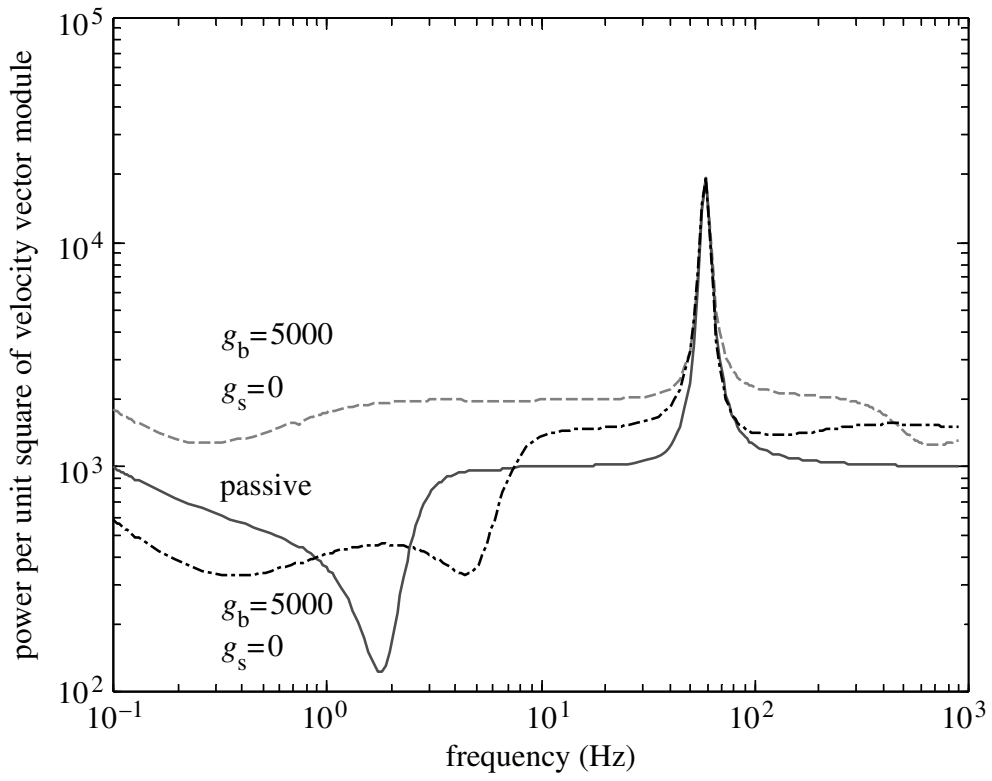

Figure 16. Effect of active control on the power per unit square of velocity vector norm $P /|\tilde{\boldsymbol{V}}|^{2}$ in the non-symmetric damping system due to different control gains $g_{\mathrm{s}}, g_{\mathrm{b}}(\eta=0.01)$.

seat suspension system $\left(g_{\mathrm{s}}=0=g_{\mathrm{b}}\right)$. The predictions show how power varies with changes to the feedback gain values assumed in different controllers. The active feedback controllers produce additional active damping and therefore the dissipation of the vibration power in the active system is increased over a significant region of the frequency band compared with the passive system. It is also observed that the seat velocity feedback control $g_{\mathrm{s}}$ provides better control than the base feedback control $g_{\mathrm{b}}$. Further calculations demonstrate that the lower bound is not affected by frequency and the larger the control gain $g_{\mathrm{s}}$, the larger the power dissipated. This suggests a way to adjust actively, in a prescribed manner, the system's damping distributions to increase vibration energy dissipation. 

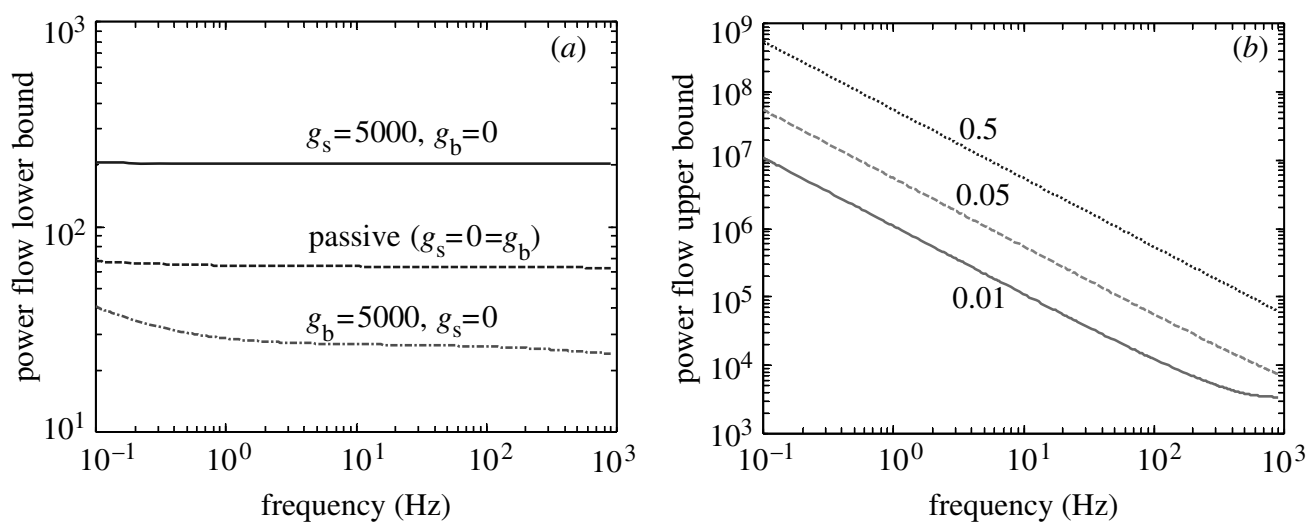

Figure 17. Comparison of power flow bound: $(a)$ lower bound influenced by active feedback control gains $g_{\mathrm{s}}, g_{\mathrm{b}} ;(b)$ upper bound influenced by beam damping $\eta$.

Figure 17 shows the influence of active and passive damping on the power flow bounds. It is seen from figure $17 a$ that the lower bound level (calculated by equation (3.16)) increases for seat velocity feedback control $g_{\mathrm{s}}=5000$, but decreases for a base velocity feedback control $g_{\mathrm{b}}=5000$ in comparison to passive isolation control findings. However, further calculations show that these active feedback controls have no effect on the upper bound value. In fact, the upper bound for this coupled system is mainly controlled by the beam's damping property. As shown in figure $17 b$, for increasing $\eta$ value from 0.01 to 0.05 , the upper bound increases up to five times of the previous bound value. This again provides an approach to increase vibration energy dissipation by increasing the upper power bound through utilizing appropriate material damping.

\section{Application of power flow design approaches}

\section{(a) Model of a suspension system}

Figure 18 illustrates a suspension system used to demonstrate application of the energy flow design and control method based on the power flow mode theory.

The difference between this system and the one shown in figure 1 is that the two dampers with damping coefficients $c_{1}$ and $c_{2}$ are arranged at arbitrary positions $l_{1}$ and $l_{2}$ measured from the left end of the bar. The two non-dimensional parameters $L_{1}=l_{1} / L$ and $L_{2}=l_{2} / L$ are adopted in the following equations.

\section{(i) Dynamic equation}

The dynamic equation of this system is given by

$$
\begin{aligned}
& {\left[\begin{array}{ll}
m L / 3 & m L / 6 \\
m L / 6 & m L / 3
\end{array}\right]\left\{\begin{array}{l}
\ddot{z}_{1} \\
\ddot{z}_{2}
\end{array}\right\}+\left[\begin{array}{ll}
c_{11} & c_{12} \\
c_{21} & c_{22}
\end{array}\right]\left\{\begin{array}{l}
\dot{z}_{1} \\
\dot{z}_{2}
\end{array}\right\}+\left[\begin{array}{cc}
k_{1} & 0 \\
0 & k_{2}
\end{array}\right]\left\{\begin{array}{l}
z_{1} \\
z_{2}
\end{array}\right\}} \\
& \quad=\left\{\begin{array}{l}
\tilde{F}_{1} \\
\tilde{F}_{2}
\end{array}\right\}=\left\{\begin{array}{l}
\tilde{f}_{1} \\
\tilde{f}_{2}
\end{array}\right\} \mathrm{e}^{\mathrm{i} \omega t},
\end{aligned}
$$




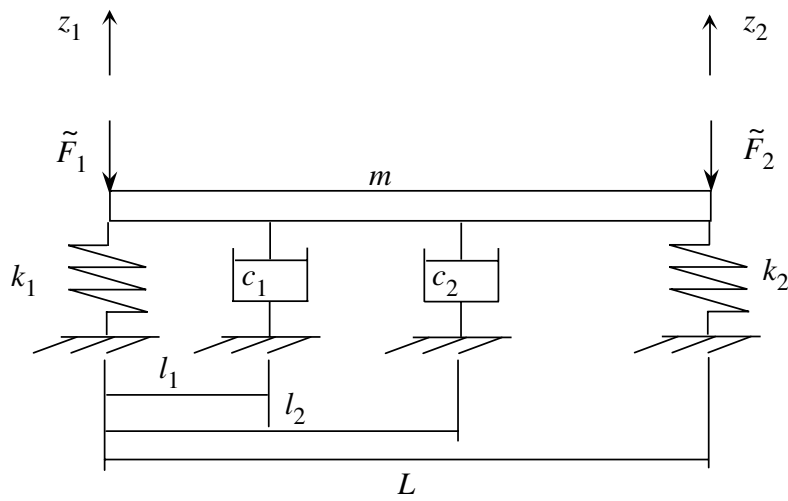

Figure 18. A simple illustrative suspension system of two degrees of freedom with two dampers mounted at arbitrary positions under the rigid bar.

where the characteristic-damping matrix $\overline{\boldsymbol{C}}$ of the system is derived from equation (3.7) in the form

where

$$
\overline{\boldsymbol{C}}=\boldsymbol{C}=\boldsymbol{a}^{\mathrm{T}}\left[\begin{array}{cc}
c_{1} & 0 \\
0 & c_{2}
\end{array}\right] \boldsymbol{a}=\left[c_{i j}\right],
$$

$$
\boldsymbol{a}=\left[\begin{array}{cc}
1-L_{1} & L_{1} \\
1-L_{2} & L_{2}
\end{array}\right] \text {. }
$$

The element $c_{i j}$ of this characteristic-damping matrix $\bar{C}$ is calculated by the following equation

$$
c_{i j}=\sum_{s=1}^{2} a_{s i} c_{s} a_{s j} \quad(i, j=1,2) .
$$

Equation (7.2) is rewritten as

$$
\overline{\boldsymbol{C}}=c_{1}\left[\begin{array}{cc}
\left(1-L_{1}\right)^{2} \pm\left(1-L_{2}\right)^{2} & L_{1}\left(1-L_{1}\right) \pm L_{2}\left(1-L_{2}\right) \\
L_{1}\left(1-L_{1}\right) \pm L_{2}\left(1-L_{2}\right) & L_{1}^{2} \pm L_{2}^{2}
\end{array}\right]
$$

for the two special cases $c_{2}= \pm c_{1}$.

(ii) Characteristic-damping factor and power flow mode

The eigenvalue problem of the characteristic-damping matrix $\overline{\boldsymbol{C}}$ is defined as

$$
\bar{C} \varphi=\lambda \varphi
$$

with characteristic equation

$$
\left|\begin{array}{cc}
c_{11}-\lambda & c_{12} \\
c_{21} & c_{22}-\lambda
\end{array}\right|=0
$$

The solutions of equation (7.6) give the characteristic-damping factors of the system

$$
\lambda_{1,2}=\frac{1}{2}\left[\left(c_{11}+c_{22}\right) \pm \sqrt{\left(c_{11}-c_{22}\right)^{2}+4 c_{12}^{2}}\right] .
$$


The corresponding power flow modes are represented by

$$
\varphi_{i}=\left[\begin{array}{c}
\alpha_{i} \\
\beta_{i}
\end{array}\right] \quad(i=1,2) .
$$

These satisfy the normalized relation used in equation $(3.8 a-c)$ and are derived by solving equation (7.5). That is

$$
\left[\begin{array}{cc}
c_{11}-\lambda_{i} & c_{12} \\
c_{21} & c_{22}-\lambda_{i}
\end{array}\right]\left[\begin{array}{c}
\alpha_{i} \\
\beta_{i}
\end{array}\right]=0 .
$$

(iii) Trace of $\overline{\boldsymbol{C}}$

The trace of the characteristic-damping matrix $\bar{C}$ is obtained as

$$
\operatorname{tr} \overline{\boldsymbol{C}}=c_{1}\left(2 L_{1}^{2}-2 L_{1}+1\right)+c_{2}\left(2 L_{2}^{2}-2 L_{2}+1\right) .
$$

(iv) Natural frequencies and modes

The physical velocity response of the system is expressed as

$$
\left\{\begin{array}{l}
\dot{z}_{1} \\
\dot{z}_{2}
\end{array}\right\}=\tilde{\boldsymbol{V}} \mathrm{e}^{\mathrm{i} \omega t}
$$

For the case of $k_{1}=k_{2}$, the natural frequencies and the corresponding natural modes of the system are calculated as follows.

Mode 1:

$$
\begin{aligned}
\omega_{1}^{2} & =2 k_{1} / m L, \\
\psi_{1} & =\left[\begin{array}{c}
\sqrt{2} / 2 \\
\sqrt{2} / 2
\end{array}\right] .
\end{aligned}
$$

Mode 2:

$$
\begin{gathered}
\omega_{2}^{2}=12 k_{1} / m L, \\
\psi_{2}=\left[\begin{array}{c}
-\sqrt{2} / 2 \\
\sqrt{2} / 2
\end{array}\right] .
\end{gathered}
$$

(v) Mode control factor

By using equations $(4.7),(7.8),(7.12 a, b)$ and $(7.13 a, b)$, we express the mode control factors of the system as

$$
\gamma_{j i}=\frac{\sqrt{2}}{2}\left[\beta_{j}+(-1)^{i+1} \alpha_{j}\right] \quad(i, j=1,2) .
$$

(b) Damping distribution design

\section{(i) Maximum $\operatorname{tr} \overline{\boldsymbol{C}}$}

Based on theorem 4.2, the necessary condition for maximum energy dissipation is that $\operatorname{tr} \overline{\boldsymbol{C}}$ is a maximum value. For the given dampers, we now examine arrangement of their positions to obtain the maximum $\operatorname{tr} \bar{C}$ and thus the maximum energy dissipation for unit power flow response $\tilde{q}_{j}$, i.e. $\left|\tilde{q}_{j}\right|^{2}=1$. 


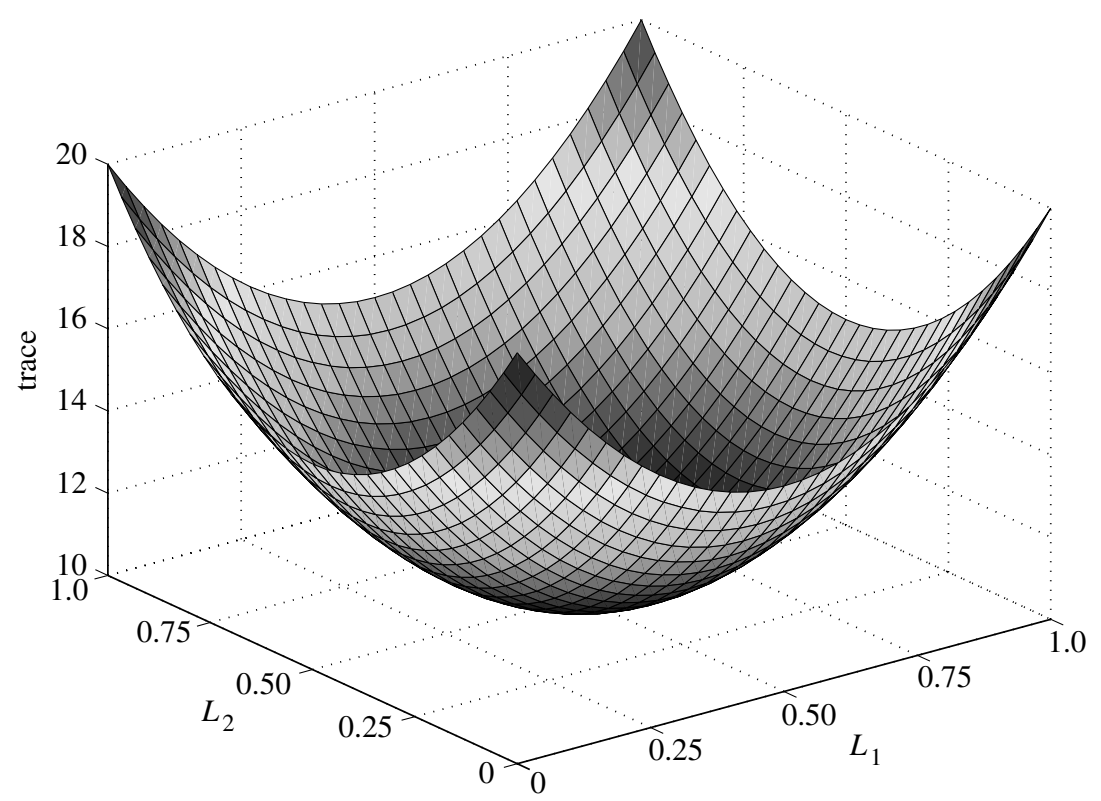

Figure 19. The value of $\operatorname{tr} \overline{\boldsymbol{C}}$ varies as a function of the position parameters $L_{1}$ and $L_{2}$.

Figure 19 shows the $\operatorname{tr} \overline{\boldsymbol{C}}$ values as a function of the position parameters $L_{1}$ and $L_{2}$. From this figure it is observed that the position of the minimum value of $\operatorname{tr} \overline{\boldsymbol{C}}$ is at the point $\left(L_{1}, L_{2}\right)=(0.5,0.5)$ and its maximum value is at the four points $\left(L_{1}, L_{2}\right)=(0,0),(0,1),(1,0)$ and $(1,1)$.

From these findings, we conclude that to obtain maximum characteristicdamping factors the two dampers are located at the ends of the rod, which also includes the four cases. For the reverse, to obtain minimum characteristicdamping factors, the two dampers are located at the middle point of the rod.

\section{(ii) Mode or motion control}

Now we investigate the suppression or retention of a heave or pitch motion of the system using theorems 4.3-4.6.

Heave motion. Using equation (3.6), we deduce from equation $(7.12 a, b)$ that the energy dissipated by the system in heave motion is given

$$
P_{1}=\psi_{1}^{\mathrm{T}} \boldsymbol{C} \psi_{1}=c_{1}+c_{2},
$$

indicating independence of position of the dampers. However, if the two dampers are arranged non-symmetrically about the mid point of the rod, in general, the motion of the rod is not a pure heave motion. To retain validity of this equation, it is required that the two dampers are symmetrically arranged. That is

$$
L_{1}+L_{2}=1 \text {. }
$$

Figure $20 a$ shows the time averaged energy dissipation per unit velocity modulus of the system, i.e. $P_{\mathrm{m}}=P /|\tilde{\boldsymbol{V}}|^{2}$ varying with location parameters $L_{1}$ and $L_{2}$ when $c_{1}=c_{2}$ and $\tilde{f}_{1}=\tilde{f}_{2}=10 \mathrm{~N}$. This figure shows that the maximum vibration power dissipation is achieved on the line $L_{1}+L_{2}=1$ along which the velocities at both ends of the rod have the same value as demonstrated in figure $20 b$ and therefore the motion of the rod is heave as defined by equation $(7.12 a, b)$. 
(a)

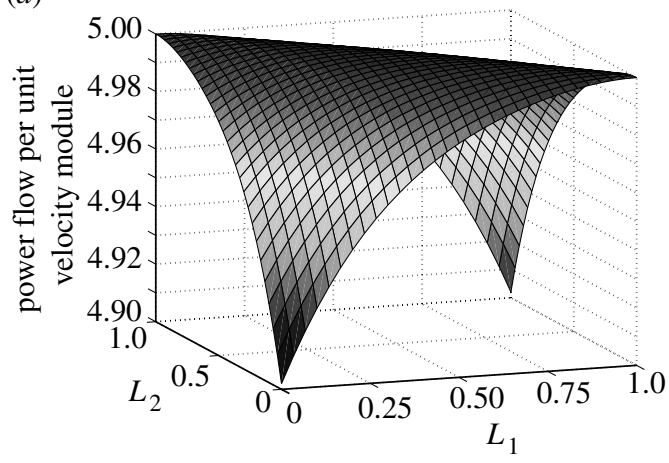

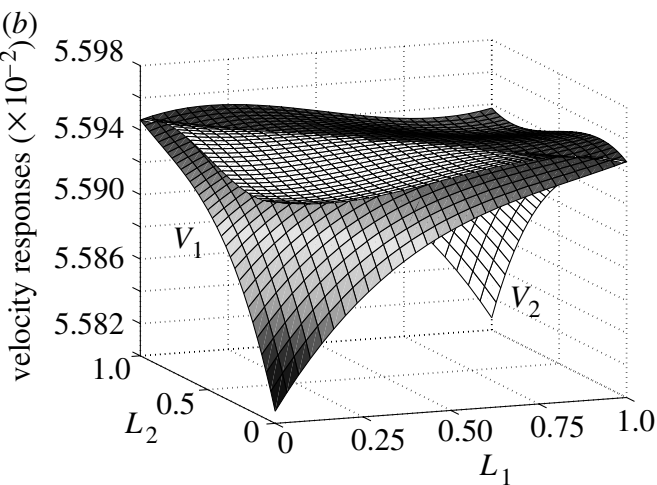

Figure 20. (a) Power flow dissipation and (b) velocities at the two ends of the rod affected by the mounting position of the two passive dampers $\left(c_{1}=c_{2}, \tilde{f}_{1}=\tilde{f}_{2}\right)$.
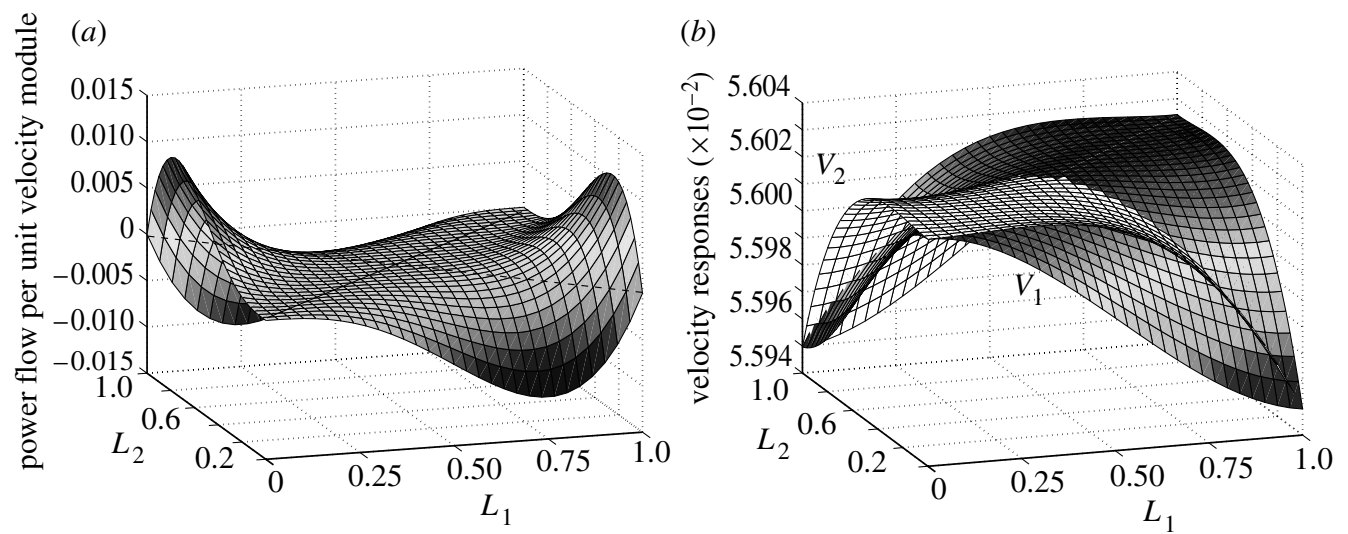

Figure 21. (a) Power flow dissipation and $(b)$ velocities at the two ends of the rod affected by the mounting position of a passive damper and an active damper satisfying equation $(7.17)\left(c_{1}=-c_{2}\right.$, $\tilde{f}_{1}=\tilde{f}_{2}$ ).

To obtain minimum energy dissipation in heave motion, an active damper with negative damping coefficient (Xing et al. 2005) is required to make

$$
P_{1}=c_{1}+c_{2}=0 \text {. }
$$

Figure 21 shows the energy dissipation of the system when an active damper satisfying equation (7.17) is applied. It is found that along the line defined by equation (7.16) and shown in figure $21 b$, there is no energy dissipation as indicated in figure 21a. Also along the line $L_{1}=L_{2}$, the energy dissipation vanishes. This is because the two dampers are arranged at the same point giving a zero resultant damping in the system.

Pitch motion. In a similar manner, the energy dissipation of the system in pitch motion described by equation $(7.3 a, b)$ is

giving

$$
P_{2}=\psi_{2}^{\mathrm{T}} \boldsymbol{C} \psi_{2}=c_{1}\left(1-2 L_{1}\right)^{2}+c_{2}\left(1-2 L_{2}\right)^{2},
$$

$$
P_{2}=\left\{\begin{array}{c}
0, \\
c_{1}=L_{2}=0.5, \\
c_{1}+c_{2}, \quad L_{1}=0,1, \quad L_{2}=0,1 .
\end{array}\right.
$$



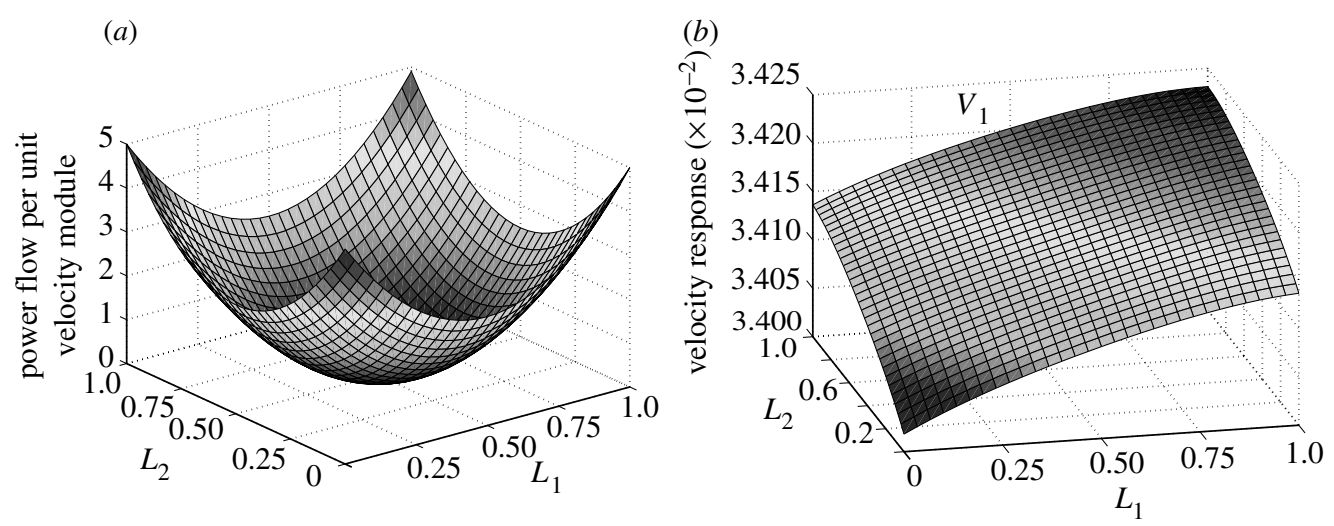

(c)

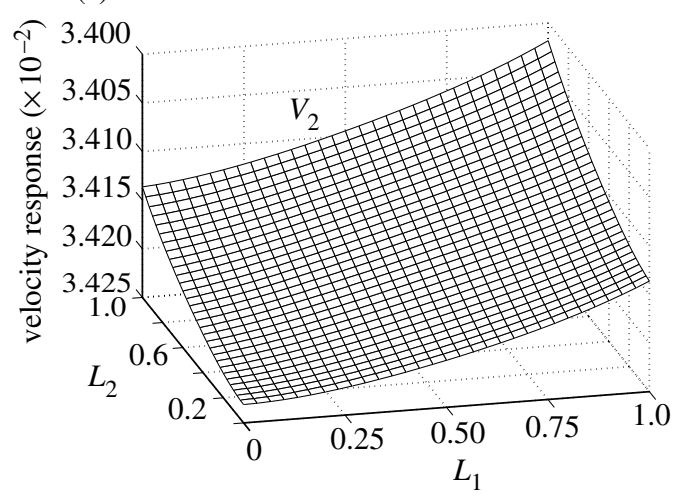

Figure 22. (a) The energy dissipation and $(b, c)$ velocities affected by the location of the two passive dampers $\left(c_{1}=-c_{2}\right)$ under excitations of $\tilde{f}_{1}=-\tilde{f}_{2}$.

To excite a pitch motion of the rod requires a pair of anti-symmetric forces $\tilde{f}_{1}=-\tilde{f}_{2}$ applied to the system together with a symmetric arrangement of the two springs and dampers.

The energy dissipated in this case is shown in figure $22 a$, and demonstrates the result given in equation (7.19). For an arrangement of two dampers located at the middle point of the rod, $L_{1}=L_{2}=0.5$, there is no power dissipated. Therefore, the pitch vibration mode is retained. However, either arranging the two dampers at the ends of the rod or both dampers at one end $\left(L_{1}=L_{2}=0\right.$ or $\left.L_{1}=L_{2}=1\right)$ or at each end $\left(L_{1}=0, L_{2}=1\right.$ or $\left.L_{1}=1, L_{2}=0\right)$, produces a maximum energy dissipation. To retain pitch motion, a symmetric arrangement of the two dampers $\left(L_{1}=0, L_{2}=1\right.$ or $L_{1}=1, L_{2}=0$ ) is appropriate. Figure $22 b, c$ shows that for this case the velocities at the two ends of the rod satisfy the pitch motion $V_{1}=-V_{2}$.

\section{Conclusions}

A generalized power flow mode theory, based on the inherent characteristics of the system's damping distribution, is developed to describe natural power flow behaviour of dynamic systems. The system's characteristic-damping matrix is constructed and the eigenvalues and eigenvectors of this matrix identify the natural 
characteristics of power flow and allow insight into energy dissipation mechanisms of the system. The proposed theoretical mathematical model describes the timeaveraged power flow in terms of system's damping and velocity response (equation (3.6)), which straightforwardly reveals vibration energy dissipation mechanisms of a dynamic system. Furthermore, in the defined power flow space, the time-averaged power flow is represented as the sum of all independent mode power components (equation (3.11)). A power flow bound theorem is proposed to approximate the upper and lower bounds of the time-averaged power. Regardless of the complexity of the dynamic system, its total time averaged power dissipation $P /|\tilde{\boldsymbol{V}}|^{2}$, as defined in equation (3.16), is bounded by one half of the lowest and highest characteristicdamping factors. This average is determined only by the damping properties of the system and is independent of any external excitations, the distributions of stiffness and mass and responses of the system.

Based on the proposed power flow mode theory, power flow design and control approaches are developed to control energy flow dispassion levels and patterns satisfying vibration control requirements. This is achieved by designing passive/active damping distributions to adjust the values of the characteristicdamping factor and mode control factors. The mode control factor reveals the coherence of a natural vibration mode or a motion form with a power flow mode. Theorems applicable for power flow design are presented in $\$ 4$ and it is demonstrated that the maximum trace of the characteristic-damping matrix of the system is a necessary condition to obtain the maximum average power dissipation. A particular vibration mode or motion form can be suppressed or retained through modifying the mode control factor by adjusting the damping distribution.

The success of the described theoretical model and power flow design approaches is demonstrated in the analysis of a range of practical examples demonstrating its applicability. The developed theory and design approaches can be widely used for modelling, analysis, design and assessment of various complex solid, fluid and acoustic systems in engineering.

\section{References}

Bishop, R. E. D. \& Price, W. G. 1979 Hydroelasticity of ships. Cambridge: Cambridge University Press.

Cuschieri, J. M. 1990 Vibration transmission through periodic structures using a mobility power flow approach. J. Sound Vib. 143, 65-74.

Fahy, F. J. \& Price, W. G. (eds) 1999 IUTAM symposium on statistical energy analysis. Dordrecht: Kluwer Academic.

Fuller, C. R., Elliott, S. J. \& Nelson, P. A. 1997 Active control of vibration. London: Academic Press.

Goyder, H. G. D. \& White, R. G. 1980 Vibrational power flow from machines into built-up structures. J. Sound Vib. 68, 59-117.

Ji, L., Mace, B. R. \& Pinnington, R. J. 2003 A mode power approach to estimating vibrational power transmitted by multiple sources. J. Sound Vib. 265, 387-399.

Langley, R. S. 1990 Analysis of power flow in beams and frameworks using the direct-dynamic stiffness method. J. Sound Vib. 136, 439-452.

Miller, D. W., Hall, S. R. \& von Flotow, A. H. 1990 Optimal control of power flow at structural junctions. J. Sound Vib. 140, 475-497. 
Moorhouse, A. T. 2002 A dimensionless mobility formulation for evaluation of excitation. J. Acoust. Soc. Am. 112, 792-980.

Nering, E. D. 1963 Linear algebra and matrix theory. London: Wiley.

Newland, D. E. 1975 An introduction to random vibrations and spectral analysis, 2 nd edn. London: Longman.

Pan, J., Pan, J. Q. \& Hansen, C. H. 1992 Total power flow from a vibrating rigid body to a thin panel through multiple elastic mounts. J. Acoust. Soc. Am. 92, 895-907.

Petersson, B. A. T. \& Plunt, J. 1982 On effective mobilities in the prediction of structure-borne sound transmission between a source and a receiver structure. Part 1. Theoretical background and basic experimental studies. J. Sound Vib. 82, 517-529.

Pinnington, R. J. 1986 Using the envelope of the frequency response in the measurement of power absorbed by a finite structure. J. Sound Vib. 109, 127-139.

Pinnington, R. J. \& White, R. G. 1981 Power flow through machine isolators to resonant and nonresonant beam. J. Sound Vib. 75, 179-197.

Price, W. G. \& Bishop, R. E. D. 1974 Probabilistic theory of ship dynamics. London: Chapman \& Hall.

Price, W. G. \& Keane, A. J. (eds) 1994 Statistical energy analysis. Phil. Trans. R. Soc. A 346(1681).

Rahnejat, H. 1998 Multi-body dynamics, vehicles, machines and mechanisms. London: Professional Engineering Publishing.

Skudrzyk, E. 1968 Simple and complex vibratory systems. University Park, PA: The Pennsylvania State University Press.

Su, J., Moorhouse, A. T. \& Gibbs, B. M. 1995 Towards a practical characterization for structureborne sound sources based on mobility techniques. J. Sound Vib. 185, 737-741.

Timoshenko, S. P., Young, D. H. \& Weaver, W. 1974 Vibration problems in engineering, 4th edn. New York: Wiley.

Xing, J. T. \& Price, W. G. 1999 A power-flow analysis based on continuum dynamics. Proc. R. Soc. A 455, 401-436.

Xing, J. T., Price, W. G. \& Wang, Z. H. 2002 A study of power flow characteristics using a vector field analysis approach. In Proc. 5th Int. Conf. on Vibration Engineering (ed. H. Hu), pp. 33-40. Beijing: China Aviation Industry Press.

Xing, J. T., Xiong, Y. P. \& Price, W. G. 2005 Passive-active vibration isolation systems to produce zero or infinite dynamic modulus: theoretical and conceptual design strategies. J. Sound Vib. 286, 615-636.

Xiong, Y. P. 1996 Power flow transmission influenced by vibration source impedance in a compound system. Chinese J. Acoust. 15, 314-318.

Xiong, Y. P., Xing, J. T. \& Price, W. G. 2000 Hybrid active and passive control of vibratory power flow in flexible isolation systems. Shock Vib. 7, 139-148.

Xiong, Y. P., Xing, J. T. \& Price, W. G. 2001 Power flow analysis of complex coupled systems by progressive approaches. J. Sound Vib. 239, 275-295.

Xiong, Y. P., Xing, J. T. \& Price, W. G. 2003a A general linear mathematical model of power flow analysis and control for integrated structure-control systems. J. Sound Vib. 267, 301-334.

Xiong, Y. P., Xing, J. T. \& Price, W. G. $2003 b$ Active vibration control of a human body-seat-boatwater dynamic interaction system excited by progressive waves. In Proceedings of 8th International Conference on Recent Advances in Structural Dynamics (ed. M. J. Brennan et al.), Paper no. 11 (CD-Rom). Southampton, UK: ISVR.

Xiong, Y. P., Xing, J. T. \& Price, W. G. 2004 A power flow mode theory based on inherent characteristics of damping distributions in systems and its applications. In Mechanics of the 21st century. Proc. 21st Int. Congress on Theoretical and Application Mechanics, ICTAM 2004, IUTAM, Warszawa, Poland (ed. W. Gutkowski \& A. T. Kowalewski), Paper No. SM25S_11834. Dordrecht: Kluwer.

As this paper exceeds the maximum length normally permitted, the authors have agreed to contribute to production costs. 